CERN-TH/96-190

\title{
The Electroweak Phase Transition through the Renormalization Group
}

\author{
N. Tetradis \\ CERN, Theory Division, \\ CH-1211, Geneva 23, Switzerland
}

\begin{abstract}
We study the high-temperature phase transitions for the Abelian and $S U(2)$ Higgs models, using the exact renormalization group. The evolution equation for a properly-defined coarse-grained free energy is solved. The phase diagram of the Abelian Higgs model has a region of second-order phase transitions governed by fixed points, for which we calculate critical exponents and crossover curves. It also has a region of first-order phase transitions, which we discuss in terms of the coarsegrained free energy. The $S U(2)$ Higgs model for small Higgs field masses has only first-order phase transitions. We derive their properties and compare with lattice and perturbative results. For larger Higgs field masses the strength of the first-order phase transitions diminishes. For masses larger than $80-100 \mathrm{GeV}$ we find evidence that they are replaced by analytical crossovers.
\end{abstract}

CERN-TH/96-190

August 1996

\footnotetext{
${ }^{1}$ E-mail: tetradis@mail.cern.ch.
} 


\section{Introduction}

The restoration of the electroweak symmetry at temperatures of the order of $100 \mathrm{GeV}$ is an important element of the Big Bang cosmology [1]. Its most prominent observable consequence is related to the possibility of generating the baryon asymmetry of the Universe during the electroweak phase transition [2]. The precise determination of the created baryon number, which could be compared with the observed matter density of the Universe, depends very sensitively on the details of the phase transition. This fact has instigated numerous studies of its characteristics during the last few years. (For recent reviews with an extensive list of references, see refs. [3, 44.) Most of the older calculations employed the perturbative approach for the determination of the temperature-dependent effective potential [5], from which the properties of the phase transition can be inferred. This approach has been pursued up to two loops [6]. However, it has been known for a long time [7] that the perturbative expansion breaks down near and in the symmetric phase of gauge theories. The reason is that at non-zero temperature the relevant expansion parameter is $\bar{e}^{2} T / m_{A}(T)$. Here $\bar{e}$ is the bare gauge coupling, and $m_{A}(T)$ the total thermal mass of the gauge field, accounting for possible thermal screening effects. The problem is due to the fact that no thermal corrections are expected for the mass of the transverse components of the gauge field within perturbation theory. This causes the perturbative expansion for the electroweak theory to diverge for small Higgs field expectation values, for which the zero-temperature mass of the gauge field is small. In order to overcome this difficulty, alternative approaches have been followed. Gap equations (truncated versions of Schwinger-Dyson equations) [8, 9] have been employed in order to obtain systematic resummations of infinite subclasses of perturbative contributions. The $\epsilon$-expansion [10] has also been used in order to obtain insight into the non-perturbative character of the phase transition. The most reliable quantitative results have been obtained through the lattice approach [11, 12, 13]. However, the underlying dynamics which results in a certain physical behaviour is often obscured by the Monte-Carlo simulations. The analytical approaches offer a more intuitive understanding. Also, the requirement of long computer time for the simulations means that the exploration of the full phase diagram for a particular model is often a formidable task.

The purpose of this paper is to present a different approach based on the exact renormalization group [14]. We employ the method of the effective average action $\Gamma_{k}$ [15], which is a generalization in the continuum of the blockspin action [16]. It results from the integration of fluctuations with characteristic momenta larger than a given scale $k$ (corresponding to the inverse of the blockspin size). The scale $k$ acts as an effective infrared regulator and gives us control over the regions in momentum space from which divergences are expected to arise in perturbation theory. The dependence of $\Gamma_{k}$ on $k$ is described by an exact renormalization-group equation [17, 18], typical of the Wilson approach to the renormalization group [14] $\mathrm{H}$. For large values of $k$ (of the order of the ultraviolet cutoff $\Lambda$

\footnotetext{
${ }^{2}$ For other versions of exact renormalization-group equations see ref. [19], and for related analyses of such equations see ref. [20].
} 
of the theory) the effective average action is equal to the classical action (no fluctuations are integrated out), while for $k \rightarrow 0$ it becomes the standard effective action (all fluctuations are integrated out). As a result, the solution of the exact renormalization-group equation, with the classical action as initial condition, gives all the physically relevant information for the renormalized theory at low scales. The formalism is constructed in Euclidean space, which makes the consideration of temperature effects straightforward, through the imposition of periodic boundary conditions in the time direction. This approach has been used in the past for the discussion of the phase transition for the $O(N)$ symmetric pure scalar theory [21, 22, 23. The perturbative study of the phase transition for this theory runs into infrared problems very similar to those for the gauge theory. Higher orders in the perturbative expansion involve powers of the quantity $\bar{\lambda} T / m_{s}(T)$ (with $\bar{\lambda}$ the bare quartic coupling and $m_{s}(T)$ the temperature-dependent mass), which diverges near the critical temperature. This problem was resolved in refs. [21, 22, 23]. The evolution of the potential as a function of $k$ was studied, using a polynomial approximation for its dependence on the field expectation value. It was shown that there are no divergent quantities in the true physical picture. The temperature-dependent quartic coupling $\lambda(T)$ is strongly renormalized near the critical temperature, so that quantities such as $\lambda(T) T / m_{s}(T)$ always stay finite. A detailed picture of the second-order phase transition emerged, with a complete mapping of the phase diagram, calculation of critical temperature and exponents, etc. The same method was subsequently applied to twoscalar theories [24, 25, 26], where again it gave a reliable, detailed picture of the more complicated phase diagram, with first-order and second-order phase transitions, crossover phenomena, tricritical points, critical exponents, etc. The assumption for a polynomial dependence of the potential on the field expectation value was relaxed in ref. [27]. The numerical integration of the full partial differential equation, for the dependence on both the field and the scale $k$, was achieved through appropriate algorithms. An immediate consequence of this development was the calculation of the equation of state for the pure scalar theory [28]. The results were confirmed by fully analytical solutions in the large $N$ limit 29]. The framework for the study of gauge theories has been provided by the generalization of the method of the effective average action and the derivation of appropriate exact renormalization-group equations [18, 30, 31]. The study of the four-dimensional Abelian Higgs model [32] has reproduced the Coleman-Weinberg mechanism for radiative symmetry breaking [33]. The same model in three-dimensions was studied in ref. [34, where a polynomial form of the potential was assumed. The phase diagram and the phase transitions, which are relevant for the superconductor phase transition, were discussed in detail. Also the high-temperature phase transition for the $S U(2)$ Higgs model was discussed in ref. [35]. The potential was calculated through an evolution equation, in which the effective infrared cutoff $k$ was replaced by the Higgs field expectation value.

In this paper we discuss the Abelian and $S U(2)$ Higgs models at non-zero temperature, by starting from the exact renormalization-group equation for the effective average action. We relax the polynomial approximation of ref. [34] and keep both the scale $k$ and the Higgs field expectation value as independent variables. Bringing the renormalization- 
group equation into a manageable form that permits its solution requires various approximations, which we spell out in detail. We list the resulting sources of errors and estimate their magnitude, whenever possible. However, some of the errors are difficult to estimate. The largest source of uncertainty is introduced by our assumption for the invariants appearing in the effective average action. Our ansatz is a truncated expression that neglects higher derivative terms. A quantitative estimate of the induced error requires the comparison with more extended truncations. As a result, an intrinsic check of the accuracy of our conclusions can be obtained through extensions of this work, which incorporate more invariants in a systematic way. First attempts in this direction were made in refs. [23, 36] for scalar theories. An alternative way of checking our results is to compare them with the results of other approaches, such as perturbation theory or lattice calculations. We make these comparisons in the following sections. Despite the limitations in accuracy, our approach provides a complete and detailed picture of the phase structure of the Abelian and $S U(2)$ Higgs models. We discuss the phase diagram in terms of the effective average potential, as a function of the Higgs field expectation value and the running scale $k$. As the potential is the generating functional of all 1PI Green's functions at zero external momenta, our solutions provide information for the running of all the generalized couplings of the theory. The discussion of fixed points, which govern the second-order phase transitions, is carried out with the inclusion of both relevant and irrelevant parameters (apart from the higher derivative terms in the action). Moreover, the consideration of the full potential permits a reliable study of first-order phase transitions. The appearance of new minima and their relative stability can be investigated in detail, without extrapolations from the form of the potential at the original minimum [34, or reliance on perturbation theory. As a result, we are able to build the complete phase diagram of the Abelian and $S U(2)$ Higgs models.

Before presenting our analysis, we mention two important points, which will be encountered in the following:

a) The discussion of a first-order phase transition usually relies on the study of a nonconvex potential. The decay of unstable minima is associated either with tunnelling fluctuations through barriers or thermal fluctuations above them. However, the effective potential is expected to be a convex quantity, with no such barriers. The resolution of this paradox lies in the realization that the effective potential is convex because the tunnelling or thermal fluctuations are incorporated in it [37]. They are associated with the low-frequency modes which are integrated out at the later stages of the evolution of a quantity such as the effective average potential. A natural approach for the study of first-order phase transitions would be to separate the problem in two parts. First, the high-frequency modes are integrated out, with the possible generation of new mimima through radiative symmetry breaking. Subsequently, the decay of unstable minima is discussed with semiclassical techniques, in the non-convex potential that has resulted from the first step. This leads us to the notion of the coarse-grained free energy, which is fundamental in statistical physics. Every physical system has a characteristic length scale associated with it. The dynamics of smaller length scales is integrated out, and is 
incorporated in the parameters of the free energy one uses for the study of the behaviour at larger scales. The Wilson approach to the renormalization group provides the realization of this intuitive idea. The coarse-graining scale (corresponding to the inverse of the characteristic length scale of the system) can be identified with $k$. The potential at this non-zero scale is not necessarily convex. In this paper we provide an explicit demonstration of how such a potential can be obtained starting from the classical action of a field theory. We also find that the intuitive separation of the problem in two parts is natural for strongly first-order phase transitions, but not so for weakly first-order ones. All this is discussed in detail in section 8. The notion of coarse graining is absent in perturbation theory. This is the main reason for the non-convergence of the perturbative series near the maxima of the classical potential, and the appearance of imaginary parts in the perturbative effective potential.

b) The infrared problem near and in the symmetric phase of gauge theories is more complicated than for scalar theories. The divergence of the quantity $\bar{e}^{2} T / m_{A}(T)$ in the perturbative expansion for gauge theories is reminiscent of the divergence of $\bar{\lambda} T / m_{s}(T)$ for scalar theories. As we have mentioned, in the latter case the renormalization group approach resolves the problem, through the identification of the relevant dynamics which is associated with infrared attractive fixed points. A similar behaviour will be observed for the Abelian Higgs model in section 7. However, an additional complication is expected for asymptotically free theories, such as the $S U(2)$ Higgs model. The absence of an effective infrared cutoff for the gauge field fluctuations near and in the symmetric phase leads to the increase of the renormalized gauge coupling. When this exceeds a critical value, the emergence of a confining regime is observed [38]. This behaviour is expected near the origin of the potential, where the zero-temperature mass of the gauge field is small, and for small values of the coarse-graining scale $k$. In this region the relevant observables are associated with bound states. An appropriate parametrization of the effective average action in terms of composite operators must be introduced, which is more efficient in capturing the relevant dynamics [39]. In this work we do not consider in detail this last part of the evolution, which requires an extensive analysis of its own. Instead we follow the evolution within the parametrization of the effective average action in terms of fundamental fields, up to the point of emergence of the confining regime. The most important element of the last stage of the evolution, for our discussion, is the appearance of an expectation value for the operator $F^{2}$, with a negative contribution to the free energy density [40]. This contribution modifies the effective average potential, as it appears only near its origin. We approximate it by $-\left(c k_{\text {conf } f}\right)^{3} T$, where $k_{\text {conf }}$ is the point in the evolution where confinement sets in, and $c$ is of order one. The detailed analysis is given in section 10 .

The paper is organized as follows: In section 2, we derive the evolution equation for the potential in the Abelian Higgs model. Two derivations are presented: a rather crude but intuitive one, and a more rigorous one, with explicit discussion of the approximations introduced. In section 3, the evolution equations for the potential and the gauge coupling are cast in a form that does not depend on the running scale $k$. This makes the identifi- 
cation of fixed points straightforward. The non-zero-temperature formalism is introduced in section 4, and the dimensional reduction of the four-dimensional, non-zero-temperature theory to an effective three-dimensional, zero-temperature one is derived in section 5 . In section 6 , the problem is reformulated as a three-dimensional one, with an appropriate initial condition. In section 7, the phase diagram for the Abelian Higgs model is derived in terms of the effective average potential, which depends on the Higgs field expectation value and the running scale $k$. Fixed points and tricritical points are identified, which govern second-order phase transitions. The critical behaviour is discussed in terms of critical exponents and crossover curves. In section 8, the first-order phase transitions are studied. The notion of coarse graining is introduced, and a non-convex potential is derived, which can be employed for the study of tunnelling. Weakly first-order phase transitions are shown to be difficult to describe in terms of semiclassical estimates for the nucleation probability. In section 9, the formalism is developed for the study of the $S U(2)$ Higgs model. The presentation is brief, because the derivations are straightforward generalizations of those in sections $2-6$. The phase transitions for the $S U(2)$ Higgs model are discussed in section 10. Arguments are presented on how the first-order phase transitions turn to continuous crossovers for large masses of the Higgs field. Our conclusions are given in section 11.

\section{Evolution equation for the potential in the Abelian Higgs model}

Before presenting the rigorous derivation, it is instructive to derive the evolution equation for the potential based on an intuitive argument, along the lines of refs. [15, 21]. The one-loop effective potential for the Abelian Higgs model with $N_{c}$ complex scalars, in $d$ dimensions, in the Landau gauge, is given by the expression

$$
\begin{aligned}
U_{k}^{(1)}(\rho)=V(\rho)+\frac{1}{2}(2 \pi)^{-d} \int d^{d} q & \left\{\ln \left[P(q)+V^{\prime}(\rho)+2 V^{\prime \prime}(\rho) \rho\right]\right. \\
& +\left(2 N_{c}-1\right) \ln \left[P(q)+V^{\prime}(\rho)\right] \\
& \left.\left.+(d-1) \ln \left[P(q)+2 \bar{e}^{2} \rho\right)\right]\right\},
\end{aligned}
$$

where $V(\rho)$ is the classical potential and $\bar{e}^{2}$ the bare gauge coupling. The three terms in the r.h.s. are the contributions of the radial scalar mode, the Goldstone modes and the gauge field. We have defined the variable

$$
\rho=\frac{1}{2} \phi^{a} \phi_{a}
$$

which we frequently use in the following. Primes denote derivatives with respect to $\rho$ : $V^{\prime}(\rho)=d V / d \rho$. We assume that the above integral is regulated by an ultraviolet cutoff $\Lambda$. The inverse propagator $P(q)$ is given by $P(q)=q^{2}$ in perturbation theory. We would 
like to introduce an effective cutoff $k$ for the low frequency modes, so that the momentum integration in eq. (2.1) does not receive contributions from modes with characteristic momenta $q^{2} \ll k^{2}$. This can be achieved through the following modification of the inverse propagator:

$$
\begin{aligned}
P(q) & =\frac{q^{2}}{1-f_{k}^{2}(q)} \\
f_{k}^{2}(q) & =\exp \left(-\frac{q^{2}}{k^{2}}\right)
\end{aligned}
$$

This form of $P$ provides for an infrared cutoff, which acts like a mass term $\sim k^{2}$ for the modes with $q^{2} \ll k^{2}$, while it leaves unaffected the modes with $q^{2} \gg k^{2}$. The potential now depends on $k$, as indicated by the subscript in eq. (2.1). We would like to derive an evolution equation for the change of $U_{k}$ with the scale $k$ and follow the evolution for $k \rightarrow 0$. This will give us control over the region of the momentum integration from which infrared divergences are expected to arise in perturbation theory. For this purpose we take the logarithmic derivative with respect to $k$ and substitute $U_{k}$ for $V$ and the running gauge coupling $e^{2}(k)$ for the bare one $\bar{e}^{2}$ in the r.h.s. of eq. (2.1). The intuitive justification for this replacement is based on the fact that the new contributions to the momentum integration, when $k$ is lowered by a small amount $\Delta k$, come from the region $k-\Delta k<q<k$. The relevant mass terms and couplings which should appear in the evolution equation are the running ones at the scale $k$ (which for the scalar modes are related to derivatives of $U_{k}$ ) and not the bare ones. This "renormalization-group improvement" results in the evolution equation

$$
\begin{aligned}
\frac{\partial U_{k}(\rho)}{\partial t}=\frac{1}{2}(2 \pi)^{-d} \int d^{d} q \frac{\partial P}{\partial t} & \left(\frac{1}{P(q)+U_{k}^{\prime}(\rho)+2 U_{k}^{\prime \prime}(\rho) \rho}+\frac{2 N_{c}-1}{P(q)+U_{k}^{\prime}(\rho)}\right. \\
& \left.+\frac{d-1}{P(q)+2 e^{2}(k) \rho}\right)
\end{aligned}
$$

where $t=\ln (k / \Lambda)$, with $\Lambda$ identified with the ultraviolet cutoff of the theory. The above equation must be supplemented with an equation for the running of $e^{2}$

$$
\frac{d e^{2}(k)}{d t}=\beta_{e^{2}}
$$

For $k=\Lambda$ the infrared and ultraviolet cutoffs coincide, and no integration of fluctuations has taken place. This determines the initial conditions for the solution of eqs. (2.4), (2.5) as $U_{\Lambda}(\rho)=V(\rho), e^{2}(\Lambda)=\bar{e}^{2}$. In the opposite limit $k \rightarrow 0$ one recovers the effective potential $U(\rho) \equiv U_{0}(\rho)$ and the renormalized gauge coupling $e_{R}^{2} \equiv e^{2}(0)$. In this heuristic derivation we have not addressed the problem of reconciling gauge invariance and the presence of a cutoff. This question can be resolved in the context of a rigorous approach, which we describe in the next paragraph.

A rigorous derivation of eq. (2.4), with an explicit determination of the approximations involved, is given in refs. 18, 30]. We give here only a brief sketch of the derivation, 
which employs the background-field formalism. One starts with a gauge-invariant classical action $S(\chi, \bar{A}+a)$ for a complex, $N_{c}$-component, scalar field $\chi$ and fluctuations $a_{\mu}$ of the gauge field around some arbitrarily chosen background field $\bar{A}_{\mu}$. To this a gaugefixing term $S_{g f}=1 /(2 \alpha) \int d^{d} x\left(\partial^{\mu} a_{\mu}\right)^{2}$ is added. Additional gauge-invariant terms $\Delta_{k} S_{S}$, $\Delta_{k} S_{G}$ are constructed, which act as effective infrared cutoffs for the scalar and gauge fields respectively. Their detailed form is given in refs. [18, 30 and involves a cutoff function $R_{k}(q)$ closely related to the form of the effective inverse propagator of eqs. (2.3). Through the standard Legendre transformation and after the removal of the infrared cutoff terms, one obtains the effective average action $\Gamma_{k}(\phi, A, \bar{A})$. This action is invariant under simultaneous gauge transformations of $\phi$ and both gauge fields $A$ and $\bar{A}$. Physical observables computed from it should be independent of $\bar{A}$ and the gauge parameter $\alpha$. $\Gamma_{k}(\phi, A, \bar{A})$ includes the effects of the integration of modes with $q^{2}>k^{2}$. For $k=\Lambda$ it coincides with the classical action plus the gauge-fixing term, while for $k \rightarrow 0$ it reproduces the effective action in the background-field formalism. It obeys the exact renormalizationgroup equation

$$
\frac{\partial \Gamma_{k}(\phi, A, \bar{A})}{\partial t}=\frac{1}{2} \operatorname{Tr}\left\{\left(\Gamma_{k}^{(2)}(\phi, A, \bar{A})+R_{k}\right)^{-1} \frac{\partial R_{k}}{\partial t}\right\},
$$

with $\Gamma_{k}^{(2)}$ the matrix of second functional derivatives with respect to $\phi$ and $A$ at fixed $\bar{A}$. The solution of the above equation is a difficult task, because the effective average action involves an infinite number of terms, even if the classical action has only a few. The manifest preservation of the gauge symmetry in the formulation constrains the form of $\Gamma_{k}(\phi, A, \bar{A})$. However, a truncation is unavoidable in practical applications. We approximate the effective average action by

$$
\begin{aligned}
\Gamma_{k}(\phi, A, \bar{A})=\int d^{d} x\{ & \left(D^{\mu} \phi_{a}\right)^{*}\left(D_{\mu} \phi^{a}\right)+U_{k}(\rho)+\frac{1}{4} Z_{F, k} F_{\mu \nu} F^{\mu \nu} \\
& \left.+\frac{1}{2 \alpha}\left[\partial^{\mu}\left(A_{\mu}-\bar{A}_{\mu}\right)\right]^{2}\right\}
\end{aligned}
$$

with $D_{\mu}=\partial_{\mu}+i \bar{e} A_{\mu}, \bar{e}$ the bare gauge coupling and $\rho$ given by eq. (2.2). The resulting evolution equation for the effective average potential, for the gauge parameter $\alpha \rightarrow 0$, is given by eq. (2.4), with the running gauge coupling defined according to

$$
e^{2}(k)=Z_{F, k}^{-1} \bar{e}^{2}
$$

Some remarks are due at this point, concerning the nature of our approximations. In eq. (2.7) we have neglected the wave-function renormalization of the scalar field. This is expected to be a good approximation, because the anomalous dimension of the three-dimensional theory, which is relevant near the phase transition, is small $(\sim 10 \%$ or less) and gives only small quantitative corrections [34]. The higher derivative terms involving the scalar field $\phi$ are not expected to introduce significant corrections either. A solution of the evolution equation for the three-dimensional pure scalar theory at the same truncation level leads to an accurate determination of the equation of state [28]. 
The largest uncertainties in our approach stem from the neglected terms involving the gauge fields $A$ and $\bar{A}$. In particular, the assumption that the only $\bar{A}$ dependence comes through a standard gauge-fixing term is rather crude. The $\bar{A}$ dependence of $\Gamma_{k}(\phi, A, \bar{A})$ is constrained by exact identities [18, 30] (which include the generalized Ward identities [41). In general one can write

$$
\Gamma_{k}(\phi, A, \bar{A})=\bar{\Gamma}_{k}(\phi, A)+\frac{1}{2 \alpha} \int d^{d} x\left[\partial^{\mu}\left(A_{\mu}-\bar{A}_{\mu}\right)\right]^{2}+\hat{\Gamma}_{k}(\phi, A, \bar{A}) .
$$

In our truncation we have approximated the gauge-invariant part $\bar{\Gamma}_{k}(\phi, A)$ through the first three terms in the r.h.s. of eq. (2.7) and completely neglected the piece $\hat{\Gamma}_{k}(\phi, A, \bar{A})$. This seems reasonable as a first step, as we are mainly interested in the evolution of $U_{k}(\rho)$ and $e^{2}(k)$. However, for general $k$ (different from $\Lambda$ or 0 ), our assumption violates the exact identities governing the dependence on the background field. A partial compensation for the neglected terms can be obtained through the introduction of appropriate correction factors in the evolution of the invariants in $\bar{\Gamma}_{k}(\phi, A)$ [30]. These correction factors mainly influence the evolution of the gauge coupling and are given in the following section of. A quantitative estimate of the error induced by the truncated form of the effective average action requires comparison with more extended truncations, and is beyond the scope of this work. However, we shall be able to draw conclusions about the accuracy of our results through a comparison with the results of other approaches.

\section{Scale-invariant form of the evolution equations}

It is convenient to cast the evolution equation (2.4) in a form that does not depend explicitly on the scale $k$. This makes the identification of possible fixed points easier. For this reason we define the dimensionless quantities

$$
\begin{aligned}
u_{k}(\tilde{\rho}) & =k^{-d} U_{k}(\rho) \\
\tilde{\rho} & =k^{2-d} \rho \\
\tilde{e}^{2}(k) & =k^{d-4} e^{2}(k)=k^{d-4} Z_{F, k}^{-1} \bar{e}^{2} .
\end{aligned}
$$

Primes on $u_{k}$ denote derivatives with respect to $\tilde{\rho}$. We can now rewrite eq. (2.4) as

$$
\begin{aligned}
\frac{\partial u_{k}}{\partial t}= & -d u_{k}+(d-2) \tilde{\rho} u_{k}^{\prime} \\
& -v_{d} L_{0}^{d}\left(u_{k}^{\prime}+2 u_{k}^{\prime \prime} \tilde{\rho}\right)-\left(2 N_{c}-1\right) v_{d} L_{0}^{d}\left(u_{k}^{\prime}\right)-(d-1) v_{d} L_{0}^{d}\left(2 \tilde{e}^{2} \tilde{\rho}\right),
\end{aligned}
$$

where

$$
v_{d}^{-1}=2^{d+1} \pi^{d / 2} \Gamma\left(\frac{d}{2}\right)
$$

\footnotetext{
${ }^{3}$ In an alternative approach, explicit gauge invariance is not imposed for the action with a cutoff 42 . The consistency of the action when the cutoff is removed is guaranteed by making sure that generalized Ward identities are satisfied by the ansatz during the whole evolution.
} 
In the second line we recognize the contributions of the radial scalar mode, the Goldstone modes and the gauge field. In the following we shall find it more convenient to integrate numerically the evolution equation for $u_{k}^{\prime}$. This reads

$$
\begin{aligned}
\frac{\partial u_{k}^{\prime}}{\partial t}= & -2 u_{k}^{\prime}+(d-2) \tilde{\rho} u_{k}^{\prime \prime} \\
& +v_{d}\left(3 u_{k}^{\prime \prime}+2 \tilde{\rho} u_{k}^{\prime \prime \prime}\right) L_{1}^{d}\left(u_{k}^{\prime}+2 u_{k}^{\prime \prime} \tilde{\rho}\right)+\left(2 N_{c}-1\right) v_{d} u_{k}^{\prime \prime} L_{1}^{d}\left(u_{k}^{\prime}\right) \\
& +2(d-1) v_{d} \tilde{e}^{2} L_{1}^{d}\left(2 \tilde{e}^{2} \tilde{\rho}\right)
\end{aligned}
$$

The dimensionless functions $L_{n}^{d}(w)$ in the above equations are given by

$$
\begin{aligned}
L_{n}^{d}(w) & =-n k^{2 n-d} \pi^{-\frac{d}{2}} \Gamma\left(\frac{d}{2}\right) \int d^{d} q \frac{\partial P}{\partial t}(P+w)^{-(n+1)} \\
& =-n k^{2 n-d} \int_{0}^{\infty} d x x^{\frac{d}{2}-1} \frac{\partial P}{\partial t}(P+w)^{-(n+1)}
\end{aligned}
$$

with $x=q^{2}$ and $P$ defined in eqs. (2.3). They have been discussed extensively in refs. 15, 23, 37] (for various forms of the infrared-regulating function). Their most interesting property, for our discussion, is that they fall off for large values of $w$, following a power law. As a result they introduce a threshold behaviour for the contributions of massive modes to the evolution equations. The various contributions to the evolution equations involve $L_{n}^{d}$ integrals with the mass eigenvalues divided by $k^{2}$ as their arguments. When the running squared mass of a massive mode becomes much larger than the scale $k^{2}$ (at which the system is probed), these contributions vanish and the massive modes decouple. We evaluate the integrals $L_{n}^{d}(w)$ numerically and use numerical fits for the solution of the evolution equations.

The last remaining element that we need is the evolution equation for the gauge coupling. For the truncation that we are using, this equation can be inferred from the results of ref. [30] and reads

$$
\frac{d \tilde{e}^{2}}{d t}=(d-4) \tilde{e}^{2}+\frac{4}{3} v_{d} N_{c} \tilde{e}^{4} l_{g c}^{d}
$$

The constant $l_{g c}^{d}$ incorporates a correction factor which partially compensates for the crude treatment of the background-field dependence in our truncation (see discussion at the end of the last section). Its values in four and three dimensions are

$$
l_{g c}^{4}=1, \quad l_{g c}^{3}=0.844 .
$$

Notice that our truncation neglects the $\rho$ dependence of the gauge coupling. This is the reason why the threshold functions, which normally would appear in the r.h.s. of eq. (3.6) [30], have been set equal to their value for zero argument. This means that the running masses of the various modes have been approximated by zero in eq. (3.6), and no threshold effects are expected in the evolution of the gauge coupling. However, the 
threshold effects in the evolution of the potential are fully accounted for. The threshold behaviour in the evolution of the gauge coupling will be important for our discussion of the phase transition for the $S U(2)$ Higgs model. An appropriate modification to the evolution equation will be introduced at that stage.

In the following sections we describe numerical solutions of the coupled system of equations (3.4), (3.6). We explicitly solve eq. (3.4) as a partial differential equation, making no additional approximations. An alternative approach has been followed in ref. 34]. There, eq. (3.4) is transformed into an infinite system of ordinary differential equations for the minimum of the potential and the coefficients of the Taylor expansion of the potential around it. This infinite system is truncated at a finite number of equations, which amounts to a polynomial approximation for the potential. For example, if the potential is approximated by a quartic polynomial and threshold effects are neglected, eq. (3.4) gives

$$
\begin{aligned}
& \frac{d \kappa}{d t}=(2-d) \kappa+4\left(N_{c}+1\right) v_{d} l_{1}^{d}+4(d-1) v_{d} l_{1}^{d} \frac{\tilde{e}^{2}}{\tilde{\lambda}} \\
& \frac{d \tilde{\lambda}}{d t}=(d-4) \tilde{\lambda}+4\left(N_{c}+4\right) v_{d} l_{2}^{d} \tilde{\lambda}^{2}+8(d-1) v_{d} l_{2}^{d} \tilde{e}^{4}
\end{aligned}
$$

with $l_{n}^{d}$ constants of order one, for the minimum $\kappa(k)$ of the rescaled potential and quartic coupling $\lambda(k)=u_{k}^{\prime \prime}(\kappa)$. The disadvantage of this approach lies in the imprecise treatment of logarithmic contributions to the potential, and the coarse description of the cases when a second minimum appears in the potential, leading to a first-order phase transition. Notice, however, that the effect of the wave-function renormalization of the scalar field and threshold effects in the running of the gauge coupling have been taken into account in [34], while we have neglected them here. It is interesting also to compare with the leading result from the $\epsilon$-expansion for the evolution of $\lambda$ and $\tilde{e}^{2}$ [43, 44, 10]

$$
\begin{aligned}
\frac{d \tilde{\lambda}}{d t} & =-\epsilon \tilde{\lambda}+4\left(N_{c}+4\right) v_{4} \tilde{\lambda}^{2}+24 v_{4} \tilde{e}^{4}-24 v_{4} \tilde{e}^{2} \tilde{\lambda} \\
\frac{d \tilde{e}^{2}}{d t} & =-\epsilon \tilde{e}^{2}+\frac{4}{3} v_{4} N_{c} \tilde{e}^{4}
\end{aligned}
$$

It is clear that, for small $\epsilon=4-d$, eqs. (3.10), (3.11) are in agreement with eqs. (3.9), (3.6) respectively $\left(l_{1}^{4}=l_{2}^{4}=1\right)$, apart from the last term in the r.h.s. of eqs. (3.10), which we could have also reproduced by taking into account the wave-function renormalization of the scalar field [34]. However, significant deviations in the values of the numerical coefficients are observed for $\epsilon=1$.

\section{The non-zero-temperature formalism}

In order to extend the formalism of the previous sections to non-zero temperature we need to recall that, in Euclidean formalism, non-zero-temperature $T$ results in periodic 
boundary conditions in the time direction (for bosonic fields), with periodicity $1 / T$ 45. This leads to a discrete spectrum for the zero component of the momentum $q_{0}$

$$
q_{0} \rightarrow 2 \pi m T, \quad m=0, \pm 1, \pm 2, \ldots
$$

As a consequence the integration over $q_{0}$ is replaced by a summation over the discrete spectrum

$$
\int \frac{d^{d} q}{(2 \pi)^{d}} \rightarrow T \sum_{m} \int \frac{d^{d-1} \vec{q}}{(2 \pi)^{d-1}}
$$

Another important point is that, due to the explicit breaking of Lorentz invariance by the temperature, the $A^{0}$ component of the gauge field evolves differently from the other three components. Therefore, it must be treated as a separate scalar in the truncated action of eq. (2.7), which develops its own mass term $m_{A^{0}}^{2}(k, \rho, T)$. With the above remarks in mind we can generalize our master equation (2.4) in order to take into account the temperature effects. For the temperature-dependent effective average potential $U_{k}(\rho, T)$ we obtain

$$
\begin{aligned}
\frac{\partial U_{k}(\rho, T)}{\partial t} & =\frac{1}{2}(2 \pi)^{-(d-1)} T \sum_{m} \int d^{d-1} \vec{q} \frac{\partial P}{\partial t} \times\left(\frac{1}{P(q)+U_{k}^{\prime}(\rho, T)+2 U_{k}^{\prime \prime}(\rho, T) \rho}\right. \\
& \left.+\frac{2 N_{c}-1}{P(q)+U_{k}^{\prime}(\rho, T)}+\frac{d-2}{P(q)+2 e^{2}(k, T) \rho}+\frac{1}{P(q)+m_{A^{0}}^{2}(k, \rho, T)}\right),
\end{aligned}
$$

with the implicit replacement

$$
q^{2} \rightarrow \vec{q}^{2}+4 \pi^{2} m^{2} T^{2}
$$

in eqs. (2.3) for $P$. For $k \sim \Lambda \gg T$, with $\Lambda$ the ultraviolet cutoff, the thermal corrections are negligible (see next paragraph). As a result the initial condition for the solution of eq. (4.3) is the same as for the zero-temperature case: $U_{\Lambda}(\rho, T)=V(\rho)$, with $V(\rho)$ the classical potential. The temperature-dependent effective potential [46]- 48 is obtained from $U_{k}(\rho, T)$ in the limit $k \rightarrow 0$.

We can put eq. (4.3) in a scale-invariant form analogous to eq. (3.2), by absorbing all the temperature dependence in generalized $L_{n}^{d}$ functions. They read

$$
L_{n}^{d}(w, T)=-2 n k^{2 n-d} \pi^{-\frac{d}{2}+1} \Gamma\left(\frac{d}{2}\right) T \sum_{m} \int d^{d-1} \vec{q} \frac{\partial P}{\partial t}(P+w)^{-(n+1)},
$$

where again the replacement (4.4) is assumed in $P$. Their basic properties can be established analytically. For $T \ll k$ the summation over discrete values of $m$ in the expression (4.5) is equal to the integration over a continuous range of $q_{0}$ up to exponentially small corrections. Therefore

$$
L_{n}^{d}(w, T)=L_{n}^{d}(w) \quad \text { for } T \ll k .
$$

In the opposite limit $T \gg k$ the summation over $m$ is dominated by the $m=0$ contribution. Terms with non-zero values of $m$ are suppressed by $\sim \exp \left(-(m T / k)^{2}\right)$. The leading 
contribution results in the simple expression

$$
L_{n}^{d}(w, T)=\frac{v_{d-1}}{v_{d}} \frac{T}{k} L_{n}^{d-1}(w) \quad \text { for } T \gg k,
$$

with $v_{d}$ defined in eq. (3.3). The two regions of $T / k$ in which $L_{n}^{d}(w, T)$ is given by the equations (4.6), (4.7) are connected by a small interval, in which the exponential corrections result in a more complicated dependence on $w$ and $T$. The above conclusions are verified by the numerical calculation of $L_{n}^{d}(w, T)$ [21.

\section{Dimensional reduction}

From this point on we concentrate on $d=4$. Our strategy is to solve eq. (4.3) (or its rescaled form, analogous to eq. (3.2) with $d=4$ and $L_{n}^{4}(w, T)$ replacing $L_{n}^{4}(w)$ ) for various temperatures, starting the evolution at some very high scale equal to the ultraviolet cutoff of the theory $k=\Lambda$. As the thermal corrections are negligible at this high scale and no quantum fluctuations have been integrated out yet, the initial condition is $U_{\Lambda}(\rho, T)=V(\rho)$, with $V(\rho)$ the classical potential. We distinguish three regions in the subsequent evolution, as $k$ is lowered from $\Lambda$ to zero $\left(\theta_{1}, \theta_{2}\right.$ are constants of order one, with $\left.\theta_{1}<\theta_{2}\right)$ :

a) $T / k \leq \theta_{1}$ : This is the low-temperature region where $L_{n}^{4}(w, T)$ are very well approximated by their zero-temperature value. Also Lorentz invariance is intact and the $A^{0}$ component evolves similarly to the other components of the gauge field. For these reasons we use the zero-temperature evolution equation (2.4) for $k \geq T / \theta_{1}$. For $T=0$ this region extends all the way to $k=0$.

b) $\theta_{1}<T / k<\theta_{2}$ : This is the threshold region, in which $L_{n}^{4}(w, T)$ do not have a simple analytical form. The $A^{0}$ component starts evolving independently of the other components and develops a mass term $m_{A^{0}}^{2}(k, \rho, T)$.

c) $T / k \geq \theta_{2}$ : In the high-temperature region eq. (4.7) gives

$$
L_{n}^{4}(w, T)=4 \frac{T}{k} L_{n}^{3}(w) .
$$

The three-dimensional character of the effective theory for modes with $q^{2} \ll T^{2}$ manifests itself in the appearance of the three-dimensional momentum integrals. It acquires here a precise quantitative meaning. Arguments from perturbation theory indicate that the mass term $m_{A^{0}}^{2}(k, \rho, T)$ for the $A^{0}$ component receives a thermal contribution $\sim N_{c} e_{R}^{2} T^{2}$. As a result this component soon decouples in this region and its contributions disappear from the evolution equations for the potential and the gauge coupling.

Similar regions exist for the evolution of the gauge coupling, even though they do not appear explicitly, due to our approximation, which neglects the threshold functions in the evolution of the gauge coupling. It is clear, however, that the evolution of $e^{2}$ in the low-temperature region is given by eq. (3.6) with $d=4$. We approximate the evolution in the threshold region by the same expression. In complete analogy with the discussion 
for the potential, the evolution of the gauge coupling in the high-temperature region is given by eq. (3.6) with $d=4$ and $l_{g c}^{4}$ replaced by

$$
l_{g c}^{4}(T)=4 \frac{T}{k} l_{g c}^{3}
$$

The above expression can be obtained by taking into account the threshold functions in the running of $e^{2}$ [30], considering the non-zero-temperature corrections, and then setting the masses to zero in the argument of the threshold functions. In order to be more precise we point out that we are assuming that, in the region where eq. (5.2) applies, the $A^{0}$ component of the gauge field has completely decoupled because it has developed a thermal mass. This is not strictly true at the beginning of the high-temperature region, where $k \sim T$, unless the gauge coupling or the number of scalar fields is large. Notice, however, that even for small coupling the omitted contribution from the $A^{0}$ component is proportional to the bare coupling and, therefore, small. As a result, the error induced by this approximation is expected to be much smaller than the errors generated through neglecting the anomalous dimension of the scalar field, or by the truncated form of the effective average action. In this work we do not study the evolution of the mass term $m_{A^{0}}^{2}(k, \rho, T)$. Instead, we rely on the perturbative result for the contribution of the $A^{0}$ field. We point out, however, that the explicit study of $m_{A^{0}}^{2}(k, \rho, T)$ is straightforward in an extended truncation.

In the high-temperature region we can define effective three-dimensional parameters by multiplying with appropriate powers of $T$ :

$$
\begin{aligned}
{ }^{3} U_{k}\left(\rho_{3}\right) & =\frac{U_{k}(\rho, T)}{T} \\
\rho_{3} & =\frac{\rho}{T} \\
e_{3}^{2}(k) & =e^{2}(k, T) T,
\end{aligned}
$$

and their dimensionless versions

$$
\begin{aligned}
u_{k}(\tilde{\rho}) & =\frac{{ }^{3} U_{k}\left(\rho_{3}\right)}{k^{3}}=\frac{U_{k}(\rho, T)}{k^{3} T} \\
\tilde{\rho} & =\frac{\rho_{3}}{k}=\frac{\rho}{k T} \\
\tilde{e}^{2}(k) & =\frac{e_{3}^{2}(k)}{k}=\frac{e^{2}(k, T) T}{k} .
\end{aligned}
$$

Making use of eqs. (5.1), (5.3), (5.4), and assuming the decoupling of the $A^{0}$ component of the gauge field, we can cast eq. (4.3) with $d=4$ in the form

$$
\begin{aligned}
\frac{\partial u_{k}}{\partial t}= & -3 u_{k}+\tilde{\rho} u_{k}^{\prime} \\
& -v_{3} L_{0}^{3}\left(u_{k}^{\prime}+2 u_{k}^{\prime \prime} \tilde{\rho}\right)-\left(2 N_{c}-1\right) v_{3} L_{0}^{3}\left(u_{k}^{\prime}\right)-2 v_{3} L_{0}^{3}\left(2 \tilde{e}^{2} \tilde{\rho}\right) .
\end{aligned}
$$


This is nothing but eq. (3.2) with $d=3$. We started with the evolution equation for the four-dimensional theory at non-zero temperature, and, in the high-temperature region, derived the evolution equation for an effective three-dimensional theory at zero temperature. Similarly, for the gauge coupling we find

$$
\frac{d \tilde{e}^{2}}{d t}=-\tilde{e}^{2}+\frac{4}{3} v_{3} N_{c} \tilde{e}^{4} l_{g c}^{3} .
$$

We have managed to reduce the problem to the set of eqs. (5.3)-(5.6). These equations have to be supplemented with appropriate initial conditions for the form of the potential and the value of the gauge coupling at the beginning of the high-temperature region $k=T / \theta_{2}$. Before addressing this point, we note that our results do not imply that the four-dimensional non-zero-temperature problem can always be reduced to the three-dimensional one. The evolution equation (5.5) involves threshold functions, which could switch off the evolution if the running masses of the various modes are large in the high-temperature region. Then the form of the potential at $k=0$ would be determined to a large extent by the initial conditions for the running in the high-temperature region. These initial conditions are determined by the evolution in the low-temperature and intermediate regions and depend strongly on the structure of the four-dimensional theory. The dynamics of the three-dimensional theory becomes dominant in the case of a second-order or weakly first-order transition near the critical temperature, where the running masses stay small, or if there is a strongly-coupled three-dimensional regime.

\section{The initial conditions for the three-dimensional evo- lution}

We now turn to the question of determining the initial conditions for the evolution in the effective three-dimensional region $k \leq T / \theta_{2}$. A straigtforward integration of the evolution equations would relate the potential and coupling at $k=T / \theta_{2}$ to the classical potential and bare coupling at $k=\Lambda$. However, a more physically transparent picture is obtained if we trade the classical parameters for those of the renormalized theory at zero temperature. An important simplification is provided by the fact that the evolution between $k=\Lambda$ and $k=T / \theta_{1}$ is identical for the zero and non-zero temperature theories. Moreover, at zero temperature the running of the various couplings between $k=T / \theta_{1}$ and $k=0$ is logarithmic and can be neglected for sufficiently small values of the couplings. Similarly, threshold effects in the evolution equation for the potential can be neglected, as the relevant mass terms are proportional to the couplings. This again is a minor approximation compared to the omission of terms in the truncated effective action. Relaxing this approximation is possible, through the numerical integration of the evolution [21]. However, in order to derive analytical expressions and not complicate our analysis (without significant improvement in accuracy) we neglect this logarithmic running at this stage. We shall later account for most of the resulting error by comparison with the perturbative result (see the end of this section). 
Let us consider a quartic, renormalized, zero-temperature potential. Higher terms in the potential are set equal to zero, consistently with our approximation of no running for the couplings. We parametrize it as 1

$$
U^{\prime}(\rho) \equiv U_{0}^{\prime}(\rho)=\lambda_{R}\left(\rho-\rho_{O R}\right)
$$

and we also define the renormalized gauge coupling

$$
e_{R}^{2} \equiv e^{2}(0)
$$

The zero-temperature evolution equation (3.4) with $d=4$ can be integrated between $k=0$ and $k=T / \theta_{1}$, if the running of the couplings and the threshold effects are neglected. We obtain for the $\rho$ derivative of the potential

$$
U_{T / \theta_{1}}^{\prime}(\rho, T)=\lambda_{R}\left\{\rho-\rho_{0 R}-\left[\left(2 N_{c}+2\right)+6 \frac{e_{R}^{2}}{\lambda_{R}}\right] v_{4}\left(\frac{T}{\theta_{1}}\right)^{2}\right\} .
$$

We observe that the effect of the evolution is a shift of the location of the minimum proportional to the square of the scale $T / \theta_{1}$ (a quadratic renormalization of the minimum). We can make the same approximations in order to integrate the non-zero-temperature evolution in the intermediate region between $k=T / \theta_{1}$ and $k=T / \theta_{2}$. However, information on the behaviour of the mass $m_{A^{0}}^{2}(k, \rho, T)$ for the $A^{0}$ component is required in this region. For this reason we separate the contribution coming from the $A^{0}$ field in order to obtain

$$
\begin{aligned}
U_{T / \theta_{2}}^{\prime}(\rho, T)= & U_{T / \theta_{1}}^{\prime}(\rho, T)+\lambda_{R}\left[\left(2 N_{c}+2\right)+4 \frac{e_{R}^{2}}{\lambda_{R}}\right] 2 v_{4} T^{2} I+C_{A^{0}}^{\mathrm{int}} \\
= & \lambda_{R}\left\{\rho-\rho_{0 R}-\left[\left(2 N_{c}+2\right)+4 \frac{e_{R}^{2}}{\lambda_{R}}\right] 2 v_{4} T^{2}\left(\frac{1}{2 \theta_{1}^{2}}-I\right)\right\} \\
& -2 v_{4} e_{R}^{2}\left(\frac{T}{\theta_{1}}\right)^{2}+C_{A^{0}}^{\mathrm{int}} .
\end{aligned}
$$

Here

$$
I=\int_{1 / \theta_{2}}^{1 / \theta_{1}} d y y t_{1}^{4}\left(0, \frac{1}{y}\right)
$$

with

$$
t_{1}^{4}\left(0, \frac{T}{k}\right)=\frac{L_{1}^{4}\left(0, \frac{T}{k}\right)}{L_{1}^{4}(0,0)}=2 \sqrt{\pi} \frac{T}{k} \sum_{n=-\infty}^{\infty} \exp \left\{-4 \pi^{2} n^{2}\left(\frac{T}{k}\right)^{2}\right\} .
$$

\footnotetext{
${ }^{4}$ The expression (6.1) corresponds to the non-convex outer part of the potential. The inner part is totally flat and irrelevant for the determination of the initial conditions for the three-dimensional evolution. We have also assumed that we are away from the parameter region where radiative symmetry breaking [33] generates a two-minimum structure in four dimensions. This typically happens for the range $\lambda_{R} / e_{R}^{4} \simeq 1 / 4 \pi^{2}$, which is not considered here. For a study of the Coleman-Weinberg phase transition in four dimensions, in the context of the effective average action, see ref. [32].
} 
For sufficiently small $\theta_{1}$ and large $\theta_{2}$

$$
I-\frac{1}{2 \theta_{1}^{2}}+2 \sqrt{\pi} \frac{1}{\theta_{2}} \simeq \int_{0}^{\infty} d y y\left[t_{1}^{4}\left(0, \frac{1}{y}\right)-1\right]=\frac{2 \pi^{2}}{3} .
$$

The last two terms in the r.h.s. of eq. (6.4) are parts of the thermal correction to the effective potential coming from the fluctuations of the $A^{0}$ component. As we explained in the previous section after eq. (5.2), we expect another small contribution coming from the evolution in the high-temperature region, until the complete decoupling of the $A^{0}$ field due to its thermal mass. Perturbation theory should give a good approximation for the total contribution, as no infrared problems are associated with the $A^{0}$ fluctuations, due to their non-zero thermal mass. For this reason we omit the $A^{0}$ field completely in the high-temperature region and include the perturbative result for its contribution in the initial conditions at $k=T / \theta_{2}$. This leads to

$$
\begin{aligned}
U_{T / \theta_{2}}^{\prime}(\rho, T)= & \lambda_{R}\left\{\rho-\rho_{0 R}-\left[\left(2 N_{c}+2\right)+4 \frac{e_{R}^{2}}{\lambda_{R}}\right] 2 v_{4} T^{2}\left(\frac{2 \sqrt{\pi}}{\theta_{2}}-\frac{2 \pi^{2}}{3}\right)\right\} \\
& +U_{A^{0}}^{\prime}(\rho, T),
\end{aligned}
$$

with

$$
\begin{aligned}
U_{A^{0}}^{\prime}(\rho, T) & =\frac{d}{d \rho}\left\{\frac{T^{4}}{2 \pi^{2}} \int_{0}^{\infty} d x x^{2} \ln \left(1-\exp \left[-\sqrt{x^{2}+\frac{m_{L}^{2}}{T^{2}}}\right]\right)\right\} \\
& =\frac{d}{d \rho}\left(\frac{1}{24} m_{L}^{2} T^{2}-\frac{1}{12 \pi} m_{L}^{3} T+\ldots\right),
\end{aligned}
$$

and

$$
m_{L}^{2}=2 e_{R}^{2} \rho+\frac{N_{c}}{3} e_{R}^{2} T^{2} .
$$

Equation (6.9) is the perturbative one-loop contribution of the $A^{0}$ component, whose total mass term, including the thermal Debye part, is given by eq. (6.10) [49, 50, 51]. The initial condition for the gauge coupling is given by

$$
e^{2}\left(T / \theta_{2}, T\right)=e_{R}^{2}
$$

This completes the first part of our study. We have reduced the problem to the threedimensional one for the couplings defined in eqs. (5.3), (5.4). The evolution equations are given by eqs. (5.5), (5.6). For the numerical part we find it more convenient to work with the evolution equation for $u_{k}^{\prime}$, given by eq. (3.4) with $d=3$. The initial conditions (at $k=T / \theta_{2}$ ) for the evolution in the three-dimensional regime are given by eqs. 6.8 - 6.11). Large values of $\theta_{2}$ minimize the error introduced by the approximate treatment of the $A^{0}$ component. However, $\theta_{2}$ must be taken sufficiently small, so that the full three-dimensional evolution is properly taken into account. We find that the optimal value is $\theta_{2}=1$. 
Before proceeding with our analysis, it is instructive and reassuring to check the validity of our approximations through a comparison with known results from perturbation theory. If we integrate the evolution equation (3.4) with $d=3$, neglecting the running of couplings and threshold effects, we find for the temperature-dependent potential

$$
\begin{aligned}
U^{\prime}(\rho, T) \equiv U_{0}^{\prime}(\rho, T) & =U_{T / \theta_{2}}^{\prime}(\rho, T)+\lambda_{R}\left[\left(2 N_{c}+2\right)+4 \frac{e_{R}^{2}}{\lambda_{R}}\right] v_{3} \sqrt{\pi} T^{2} \frac{1}{\theta_{2}} \\
& =\lambda_{R}\left\{\rho-\rho_{0 R}+\left[\left(2 N_{c}+2\right)+6 \frac{e_{R}^{2}}{\lambda_{R}}\right] \frac{T^{2}}{24}\right\},
\end{aligned}
$$

where we have kept only the leading term in the expansion of the $A^{0}$ contribution of eq. (6.9). This predicts a critical temperature

$$
T_{c r}^{2}=\frac{24 \rho_{0 R}}{\left(2 N_{c}+2\right)+6 \frac{e_{R}^{2}}{\lambda_{R}}},
$$

in agreement with the lowest-order perturbative result [49, 50]. Corrections to the above expression arise through the inclusion of the running of couplings and threshold effects in the evolution. Another check can be made by noticing that, if the contributions from the scalar fields and the running of couplings are neglected, eq. (4.3) can be easily integrated in order to reproduce the one-loop perturbative contribution coming from the gauge field. In our approach we have reformulated eq. (4.3) in terms of the three-dimensional evolution with an appropriate boundary condition. As a check we present in fig. 1 the results of the numerical integration of eq. (5.5), in which we have omitted the scalar contributions and the running of $e^{2}$. We have used $N_{c}=1, \lambda_{R}=0.02, e_{R}^{2}=0.09, T^{2} / \rho_{0 R} \simeq 0.953$. Line (a) is the perturbative one-loop result coming from gauge-field contributions. Line (b) results from the numerical integration of the evolution equation (5.5), if we neglect the first and second terms in the second line, which come from the scalar modes. The observed discrepancy can be attributed to the omission of the threshold corrections in the four-dimensional running, which determines the initial condition of eq. (6.8). The biggest part of the discrepancy is due to an imprecise value for the renormalization of the location of the minimum, for which we have used

$$
\Delta \rho_{0}\left(k=T / \theta_{2}, T\right)=8 \frac{e_{R}^{2}}{\lambda_{R}} v_{4} T^{2}\left(\frac{2 \sqrt{\pi}}{\theta_{2}}-\frac{2 \pi^{2}}{3}\right)=-0.32981 \rho_{0 R} .
$$

Due to the threshold effects this value receives corrections proportional to powers of the couplings $\lambda_{R}, e_{R}^{2}$. For the cases we study in the following, the largest corrections are proportional to $e_{R}^{4}$. Line (c) is the result of the numerical integration if the minimum of the above potential is shifted by an additional amount $\delta \rho_{0}\left(k=T / \theta_{2}, T\right)=-0.00082 \rho_{0 R}$ for the same critical temperature. We observe very good agreement. We can alternatively view the need for the additional shift of the minimum as a correction to the value of the critical temperature that we have used. This means that our value for the critical temperature cannot be trusted to better than $0.3 \%$. However, approximations associated 
with the truncated form of the effective average action are expected to generate even larger errors. In any case, in order to eliminate this particular source of uncertainty, we repeat the empirical procedure that we have used above for every model we study in the following. Through comparison with the perturbative result, we determine an empirical value $\delta \rho_{0}\left(T / \theta_{2}\right)$, which we incorporate in the initial condition of eq. (6.8).

\section{The phase diagram and the second-order phase transitions}

In the previous sections we developed the formalism that we need for the study of the phase transitions in the Abelian Higgs model. We reduced the problem of calculating the temperature-dependent effective potential to the question of integrating the evolution equations (5.5) (or equivalently eq. (3.4) with $d=3$ ) and (5.6), for the effective threedimensional parameters (effective average potential and running gauge coupling) defined in eqs. (5.3), (5.4). The evolution starts at the scale $k=T / \theta_{2}$ (we use $\theta_{2}=1$ ), with the initial conditions given by eqs. (6.8)-(6.11) in terms of the renormalized parameters of the zero-temperature theory. The temperature-dependent potential is obtained in the limit $k \rightarrow 0$. The initial condition for the potential incorporates the contributions from the part of the evolution that integrates out fluctuations with four-dimensional character. The remaining part is purely three-dimensional and the universal results of our study (such as the existence of fixed points) are characteristic of the three-dimensional theory. For this reason they are relevant for all theories that belong to the same universality class. For example, they can be applied to the theory of the superconductor phase transition. Contact with perturbation theory can be made if one neglects the contributions to eq. (5.5) that come from the scalar modes (the first and second terms in the second line) and the evolution of $e^{2}$ (by neglecting the second term in the r.h.s. of eq. (5.6)). The integration reproduces the part of the one-loop result that comes from the fluctuations of the gauge field. If the scalar field contributions are included, but the gauge-coupling running is still neglected, agreement with perturbation theory is not possible. The reason is that perturbation theory at a finite order cannot correctly describe the relevant infrared physics associated with massless (or almost massless) scalar modes. Resummations of infinite subclasses of perturbative diagrams through gap equations [46, 8, 9, 22] may ameliorate the situation, but even they do not predict correct values for quantities such as critical exponents. We shall return to this point in the following. The inclusion of the running of the gauge coupling generates further deviations from the perturbative results.

We start by discussing the phase diagram for the Abelian Higgs model with $N_{c}$ complex scalars. The expected qualitative behaviour can be inferred by considering the evolution equations (5.6) and (3.9) (with $d=3$ ), for the gauge and quartic couplings. We point

out that eq. (3.9) assumes a quartic form for the potential and no threshold effects, and for this reason the predicted behaviour is not trustworthy. However, it is instructive to consider this simplified system of equations first and perform the complete analysis later. The flows of couplings are depicted in fig. 2 for a theory with $N_{c}=250$. They exist on 
a critical surface, with the minimum of the potential (or the mass term) as the relevant parameter. One may consider the flows as being unstable perpendicularly to the plane of fig. 2. Moving above or below the critical surface corresponds to a growing or diminishing value for the minimum, and the respective deviations lead to the phase with symmetry breaking or the symmetric one [21, 23]. We observe the existence of three fixed points on the critical surface:

a) The Wilson-Fisher fixed point (WF) has $\tilde{e}^{2}=0$ and corresponds to the pure scalar theory. It is unstable in the $\tilde{e}^{2}$ direction.

b) The Abelian fixed point (A) is the most stable one and is expected to be relevant very close to the critical temperature of the second-order phase transitions.

c) The tricritical point $(\mathrm{T})$ separates the region of second-order phase transitions from the region of first-order ones. The latter are expected when the quartic coupling $\tilde{\lambda}$ turns negative, indicating the appearance of an instability [52, 24].

The above phase diagram ceases to exist below $\left(N_{c}\right)_{c r}=222$, where the Abelian and tricritical points disappear and all the flows end up in the region of first-order phase transitions. This value of $\left(N_{c}\right)_{c r}$ is of the same order of magnitude as the prediction from the $\epsilon$-expansion [43].

We now replace the evolution equation (3.9) with the partial differential equation (5.5) for the full potential. This equation automatically takes into account the running of all the higher couplings and the threshold effects. In practice, we find more efficient to integrate eq. (3.4) with $d=3$. Two algorithms for the numerical integration have been presented in detail in ref. [27]. The comparison of the two methods provides a good check for possible systematic numerical errors. The two algorithms give results which agree at the $0.3 \%$ level. We expect the numerical solution to be an approximation of the solution of the partial differential equation (3.4) with the same level of accuracy. In fig. 3 we present the results of the numerical integration of eq. (3.4) for $d=3$ and $N_{c}=5$. We consider a renormalized, zero-temperature potential given by eq. (6.1) with $\lambda_{R}=0.5$. The renormalized gauge coupling at zero temperature is chosen as $e_{R}^{2}=10^{-6} \times e_{A}^{2}$, where $e_{A}^{2}$ is its value at the Abelian fixed point. The temperature is very close to the critical one $T_{c r}^{2} / \rho_{0 R} \simeq 2.10$. (We use the value of the zero-temperature minimum in order to renormalize all dimensionful quantities.) The function $u_{k}^{\prime}(\tilde{\rho})$ is plotted for various values of $k$, starting from the beginning of the high-temperature region $k=T / \theta_{2}$ (dotted lines). As $k$ is lowered the system approaches first the Wilson-Fisher fixed point, and the evolution slows down around the scale-invariant solution corresponding to this fixed point (solid lines). During this part of the evolution the gauge coupling $\tilde{e}^{2}$ stays small and the theory is dominated by the scalar modes. Subsequently the gauge coupling becomes significant and acts as a relevant perturbation, which causes the system to leave the Wilson-Fisher fixed point and approach the Abelian one. Eventually $u_{k}^{\prime}(\tilde{\rho})$ moves away from the Abelian fixed point too, and the final running leads to the phase with spontaneous symmetry breaking (dashed lines). The relevant perturbation for this last deviation is the value of the minimum of the potential at the beginning of the running or, equivalently, the temperature. With sufficient fine-tuning of the temperature the system can spend a long 
"time" $t=\ln \left(k / \rho_{0 R}^{1 / 2}\right)$ in the vicinity of the fixed points. During this "time", the minimum $\kappa$ of the potential $u_{k}(\tilde{\rho})$ varies very little, while the minimum of $U_{k}(\rho, T)$ evolves towards zero according to

$$
\rho_{0}(k, T)=k \kappa .
$$

The longer $u_{k}^{\prime}(\tilde{\rho})$ stays near the fixed points, the smaller the resulting value of $\rho_{0}(k, T)$ when the system deviates from it. As this value determines the mass scale for the renormalized theory at $k=0$, the fixed-point solutions govern the behaviour of the system very close to the phase transition, where the characteristic mass scale goes to zero. As a result the value of the temperature that keeps the system close to the fixed points for an arbitrarily long "time" is the critical one. Also, the minimum of the potential and the characteristic mass scale go continuously to zero as the critical temperature is approached. This demonstrates the presence of second-order phase transitions when the behaviour of the system is governed by the fixed points. The approach to the fixed-point solutions and the deviation from them can also be seen in fig. 4. We plot the evolution of the minimum $\kappa(k)$ of $u_{k}(\tilde{\rho})$, the coupling $\tilde{\lambda}(k)=u_{k}^{\prime \prime}(\kappa)$, and the gauge coupling $\tilde{e}^{2}(k)$. All higher couplings, related to higher derivatives of $u_{k}$, can be easily obtained from the solution of fig. 3. For the solution depicted in figs. 3 and 4 the temperature is slightly below $T_{c r}$. The potential $u_{k}(\tilde{\rho})$ moves away from the Abelian fixed point and its minimum $\kappa(k)$ grows in such a way that $\rho_{0}(k, T)$ approaches a constant value for $k \rightarrow 0$. Eventually the theory settles down in the phase with spontaneous symmetry breaking with a renormalized value $\rho_{0 R}(T)$ for the minimum of $U(\rho, T)$. If the temperature is larger than $T_{c r}$, the minimum $\kappa(k)$ runs to zero and $u_{k}^{\prime}(0)$ becomes positive and subsequently increases. The system settles down in the symmetric phase with a positive constant renormalized mass term $m_{R}^{2}(T)=k^{2} u_{k}^{\prime}(0)$ as $k \rightarrow 0$. We point out that larger deviations from $T_{c r}$ cause the system to deviate towards the phase with spontaneous symmetry breaking or the symmetric one earlier in the evolution. As a result, the relative influence of the various fixed points on the renormalized theory depends sensitively on the precise value of the temperature.

Another important property of the "near-critical" trajectories, which spend a long "time" $t$ near the fixed points, is that they become insensitive to the details of the zerotemperature theory, which determine the initial conditions for the evolution in the threedimensional region. After $u_{k}^{\prime}(\tilde{\rho})$ has evolved away from its scale-invariant form near a fixed point, its shape is independent of the choice of $\lambda_{R}$ and $e_{R}^{2}$. This property gives rise to the universal critical behaviour near second-order phase transitions. As the Abelian fixed point is the most attractive one, it is expected to determine the characteristics of the phase transition very close to the critical temperature. The most typical quantities parametrizing the behaviour near the transition are the critical exponents, which are universal quantities depending only on the dimensionality of the system and its internal symmetries. We concentrate on the exponent $\nu$, which parametrizes the behaviour of the renormalized mass in the critical region. Another critical exponent is the anomalous dimension $\eta$ of the scalar field, which we have approximately taken to be zero. The other exponents are not independent quantities, but can be determined from $\eta$ and $\nu$ through universal scaling laws. We define the exponent $\nu$ through the renormalized mass term in 
the symmetric phase $m_{R}^{2}(T)=k^{2} u_{k}^{\prime}(0)$ for $k \rightarrow 0$. The behaviour of $m_{R}^{2}(T)$ in the critical region depends only on the distance from the phase transition, which can be expressed in terms of the difference of the temperature $T$ from the critical temperature $T_{c r}$, for which the renormalized theory has exactly $m_{R}^{2}\left(T_{c r}\right)=0$. The exponent $\nu$ is defined through the relation

$$
m_{R}^{2}(T) \sim\left(T^{2}-T_{c r}^{2}\right)^{2 \nu}
$$

For a determination of $\nu$ from our results we calculate $m_{R}^{2}(T)$ for various values of $T^{2}-$ $T_{c r}^{2}$. We subsequently plot $\ln \left(m_{R}^{2}\right)$ as a function of $\ln \left(T^{2}-T_{c r}^{2}\right)$ and infer an "effective" exponent $\nu$ from the slope. In fig. 5 we plot this "effective" exponent for the model of fig. 3 as the critical temperature is approached. Each point along the curve corresponds to trajectories similar to those of figs. 3 and 4 , which approach and subsequently deviate from the fixed points at various "times" $t=\ln \left(k / \rho_{0 R}^{1 / 2}\right)$. For the model of fig. 3, the initial conditions (determined by the zero-temperature theory) place the system initially close to the Wilson-Fisher fixed point. As the critical temperature is approached, the system stays longer near the fixed points before deviating towards the symmetric phase. As the Abelian fixed point is the most attractive one, it dominates the largest part of the evolution for very small $T-T_{c r}$. This happens because the gauge coupling has enough "time" to grow towards the attractive fixed point of eq. (5.6). This behaviour is reflected on the "effective" exponent $\nu$. It first approaches a value typical of the Wilson-Fisher fixed point, but very close to $T_{c r}$ it settles down at a value characteristic of the Abelian fixed point. The part of the curve between the Wilson-Fisher and the Abelian fixed points is typical of a crossover curve [24, 25]. Values of the critical exponent $\nu$ for the WilsonFisher fixed point, for various $N_{c}$, calculated through the method of the effective average action, have been presented in refs. [23, 27, 28]. In table 1 we give values characteristic of the Abelian fixed point. They are consistent with the results of ref. [34], if one takes into account the difference in the approximations employed. (We have neglected the anomalous dimension of the scalar field and used a general form for the potential, whereas in ref. [34 the anomalous dimension is taken into account, but the potential is approximated by a polynomial.) However, in contrast to ref. [34, we find that the tricritical point and the Abelian fixed point disappear for $N_{c}<5$. The two approaches are compared at the end of the section.

In order to complete the phase diagram, we must investigate the region that leads to first-order phase transitions. In the simplified picture of fig. 2 a tricritical point (T) separates the regions of first-order and second-order phase transitions. The form of the full potential at this point is presented in fig. 6, along with its form at the Wilson-Fisher (WF) and the Abelian (A) fixed points. At the tricritical point the potential develops a new unstable minimum at the origin $\left(u_{k}^{\prime}(0)\right.$ is positive). Another characteristic point is the inflection point (I), where the minimum of the potential away from the origin disappears and the origin remains the only minimum. Various patterns of evolution can produce interpolations between the different forms of the potential presented in fig. 6. A typical example was given in fig. 3. For other values of $\lambda_{R}$ and $e_{R}^{2}$ the potential may evolve first towards the tricritical point, and subsequently be attracted to the Abelian fixed 
point, before deviating towards the phase with spontaneous symmetry breaking or the symmetric one. An unstable minimum appears for part of the evolution, but eventually disappears. Flows of this type again give second-order phase transitions. For the same

$e_{R}^{2}$ and smaller $\lambda_{R}$ the flow leads from the tricritical point to the inflection one. Along the way the absolute minimum of the potential shifts from the minimum away from the origin to the origin. This typical behaviour characterizes first-order transitions. It has been observed in the past in the context of the effective average action, using cruder approximations [24, 26, 32]. We study the first-order phase transitions in detail in the next section.

We conclude this section by comparing our results with those of other approaches. Perturbation theory cannot reproduce the behaviour associated with fixed points. It cannot capture the dynamics of massless (or almost massless) scalar modes. As a result, no part of the rich structure that we presented in this section is visible to it. The leading result from the $\epsilon$-expansion is in agreement with the qualitative structure of the phase diagram of fig. 2 for a large number of scalar fields $N_{c}=\mathcal{O}(100)$ 43. In contrast, we have found that the tricritical point and the Abelian fixed point persist down to $N_{c}=5$. We believe that our conclusion gives a more reliable estimate of the order of magnitude of $\left(N_{c}\right)_{c r}$, below which the phase diagram of fig. 2 ceases to exist, because our analysis was carried out directly in three dimensions. A very important question is whether the fixedpoint structure that we discussed exists also for $N_{c}=1$. This would raise the possibility of a second-order superconducting phase transition [53]. The analysis of ref. [34] supports this possibility through the study of the same renormalization-group equation for the effective average action. The difference lies in the approximations involved. We have solved numerically the evolution equation for the full potential without using a Taylor expansion around a minimum, which was done in ref. [34. This has permitted us to investigate the appearance of a second minimum without resorting to extrapolations. We have neglected effects that are expected to be small, such as the anomalous dimension of the scalar field and threshold effects in the running of the gauge coupling. However, a major source of uncertainty in both approaches is related to the assumed truncated form of the effective average action. Effects coming from the $\rho$ dependence of the gauge coupling, higher derivative terms, and the role of the background gauge field $\bar{A}$ have not been taken into account. An extended truncation is needed in order to estimate their magnitude. At the present stage we can conclude that the phase diagram of fig. 2 survives down to a critical number of scalar fields $\left(N_{c}\right)_{c r}=\mathcal{O}(1)$. This supports the possibility of a second-order superconductor phase transition, but does not guarantee it.

\section{The first-order phase transitions}

We now turn to the detailed study of the region of first-order phase transitions. We concentrate on the case $N_{c}=1$ because it is the most relevant for physical applications, such as the non-zero-temperature behaviour of superconductors. The analysis is very similar for other values of $N_{c}$. The expected phase diagram can again be deduced from 
the set of equations (5.6) and (3.9) (with $d=3$ ), for the gauge and quartic couplings. It is depicted in fig. 7. We observe that the Abelian fixed point and the tricritical point have disappeared and all the flows end up in the region of first-order phase transitions. We now replace the evolution equation (3.9) with the partial differential equation (5.5) for the full potential. This takes into account the running of all the higher couplings and the threshold effects. In fig. 8 we present the results of the numerical integration of the evolution equation for the potential for $N_{c}=1$. We consider a renormalized, zero-temperature potential given by eq. (6.1) with $\lambda_{R}=0.5$. The renormalized gauge coupling at zero temperature is chosen as $e_{R}^{2}=10^{-7} \times e_{A}^{2}$, where $e_{A}^{2}$ is its value at the Abelian fixed point. The temperature is very close to the critical one $T_{c r}^{2} / \rho_{0 R} \simeq 6.41$. The function $u_{k}^{\prime}(\tilde{\rho})$ is plotted for various values of $k$, starting from the beginning of the hightemperature region $k=T / \theta_{2}$ (dotted lines). As $k$ is lowered the system approaches first the Wilson-Fisher fixed point, and the evolution slows down around the scale-invariant solution corresponding to this fixed point (solid lines). During this part of the evolution the gauge coupling $\tilde{e}^{2}$ stays small and the theory is dominated by the scalar modes. Subsequently the gauge coupling becomes significant and acts as a relevant perturbation, which causes the system to leave the Wilson-Fisher fixed point. In contrast to fig. 3 no other fixed points exist which could attract the flow of the potential. At some point during the evolution (dashed lines) a new minimum appears at the origin $\left(u_{k}^{\prime}(0)\right.$ turns positive). Subsequently it becomes deeper than the initial minimum, and eventually the only minimum of the potential. The behaviour at the later stages of the evolution is characteristic of a first-order phase transition. By slightly reducing the temperature we could arrange for the potential $U_{k}(\rho, T)$ to end up with two minima of equal depth for $k \rightarrow 0$. We explicitly demonstrate how this happens in the rest of this section. In fig. 9 we display the evolution of the original minimum $\kappa$ of $u_{k}(\tilde{\rho})$, the quartic coupling $\tilde{\lambda}(k)=u_{k}^{\prime \prime}(\kappa)$ and the gauge coupling $\tilde{e}^{2}(k)$. We observe how $\tilde{\lambda}(k)$ goes to zero at the point where the original minimum disappears.

The Wilson-Fisher fixed point is important only for very small values of the zerotemperature coupling $e_{R}^{2}$. We are interested in larger values of $e_{R}^{2}$ (comparable to $\lambda_{R}$ ) in order to develop some intuition relevant for the electroweak phase transition. For this range of $e_{R}^{2}$ the scale-invariant solution of the evolution equation for the potential is never approached. Similarly, the question of the persistence of the Abelian fixed point and the tricritical point down to $N_{c}=1$ (see end of last section) is not relevant. The characteristic evolution of the potential $U_{k}(\rho, T)$ as $k$ is lowered is depicted in fig. 10 . In order to produce fig. 10, we have solved numerically the evolution equation for the derivative of the rescaled potential $u_{k}^{\prime}(\tilde{\rho})$ as before, and deduced $U_{k}(\rho, T)$ through eqs. (5.3), (5.4). The zero-temperature theory is given by eq. (6.1) with $\lambda_{R}=0.02$, and $e_{R}^{2}=0.09$. The temperature is $T^{2} / \rho_{0 R} \simeq 0.841$. Initially the potential has only one minimum away from the origin, which receives a renormalization proportional to the running scale $k$ during the evolution, and moves closer to the origin. At some point a new minimum appears at the origin. It is induced by the integration of thermal fluctuations, through the generalization of the Coleman-Weinberg mechanism. The evolution slows 
down at the later stages and the potential converges towards a non-convex profile with two minima of equal depth. Around the minima the scale $k$ becomes smaller than the mass of the various massive modes, and this induces their decoupling. We have stopped the evolution at a non-zero $k_{b}$, for which the shape of the potential is most stable. The presence of the non-convex part is explained by this non-zero value of $k$. We have not yet integrated out all the thermal fluctuations, which should render the effective potential convex. More specifically, we have not taken into account fluctuations that interpolate between the two minima of fig. 10 [37]. They are the ones that trigger the thermal tunnelling and drive the first-order phase transition. If we continue the evolution all the way to $k=0$, these interpolating configurations will be gradually integrated out. As a result, the height of the barrier will start getting smaller, until the region of the potential between the two minima becomes flat.

For the model of fig. 10 the behaviour of the potential suggests the separation of the study of the first-order phase transition into two parts. First, the evolution equation is integrated down to a given scale $k_{b}$, where the shape of the potential becomes approximately stable around a non-convex form. The scale $k_{b}$ can be identified with the coarse-graining scale, at which the free energy is defined in all studies of statistical systems. The effective average action at this scale plays the role of the coarse-grained free energy. Our formalism provides the necessary framework for the derivation of the coarse-grained free energy from the bare action. This should be contrasted with the perturbative approach, in which the notion of coarse graining is absent. The second part of the problem concerns the dynamics of the first-order phase transition in terms of the well-defined potential. In the context of the nucleation picture [54, semiclassical techniques can be employed in order to estimate the nucleation probability, based on a dominant configuration that is usually referred to as the critical bubble [55. The characteristics of the critical bubble can be deduced from the form of the potential and we present them in the following.

In fig. 11 we compare various approximations of the form of the potential at the respective critical temperatures of the first-order phase transitions for the model of fig. 10. Line (a) is the perturbative one-loop result if the contribution of the scalar fluctuations is neglected. The integration of the evolution equation, if the terms involving scalar modes and the running of the gauge coupling are neglected, reproduces this result. Line (b) is the perturbative one-loop result when the scalar fluctuations are taken into account. We have used expressions analogous to eq. (6.9), with thermally corrected masses given by

$$
\begin{aligned}
& m_{G}^{2}=\lambda_{R} \rho+\left(\frac{1}{4} e_{R}^{2}+\frac{2 N_{c}+2}{24} \lambda_{R}\right)\left(T^{2}-\frac{24 \rho_{0 R}}{\left(2 N_{c}+2\right)+6 \frac{e_{R}^{2}}{\lambda_{R}}}\right) \\
& m_{R}^{2}=3 \lambda_{R} \rho+\left(\frac{1}{4} e_{R}^{2}+\frac{2 N_{c}+2}{24} \lambda_{R}\right)\left(T^{2}-\frac{24 \rho_{0 R}}{\left(2 N_{c}+2\right)+6 \frac{e_{R}^{2}}{\lambda_{R}}}\right)
\end{aligned}
$$

for the Goldstone and radial modes, respectively. Due to the smallness of the quartic coupling $\lambda_{R}$, the effect of the scalar fluctuations is minor. Line (c) results from the 
integration of the full evolution equation for the potential down to the scale $k_{b}$, if the running of $e^{2}$ is neglected. We observe that the predicted strength of the first-order phase transition is much smaller than what is expected from one-loop perturbation theory (line (b)). This is due to the inability of perturbation theory to properly describe the infrared dynamics of massless (or almost massless) scalar fluctuations. The same problem has also been encountered in the study of the phase transition for the pure scalar theory. Perturbation theory at any finite order predicts a first-order phase transition for this system [56]. The resummation of an infinite subclass of perturbative diagrams through gap equations 46, 8, 9, 22] improves the situation, but only the use of the renormalization group [21] gives the full picture of a second-order phase transition characterized by critical exponents. Line (d) results from the integration of the evolution equation for the potential with the running of $e^{2}$ included. The theory is infrared-free and the running gauge coupling gets smaller when $k$ is reduced. As the first-order phase transition is triggered by the gauge field fluctuations, its strength is reduced because the "effective" gauge coupling is smaller than $e_{R}^{2}$. We should also mention that a reduction of the strength of the phase transition is observed in higher orders of perturbation theory, where the running of the couplings is partly accounted for [50].

The characteristics of the phase transition can be deduced from the form of the potential. The most natural scale for the determination of the various parameters is the coarse-graining scale $k_{b}$. This is convenient, because the masses of various fields are often renormalized to zero for $k \rightarrow 0$, due to approximations or the presence of massless modes in the evolution. For example, the running of the gauge coupling (which determines the mass of the gauge field) never stops, due to the omission of threshold effects in the evolution equation (5.6). Also the running of the quartic coupling at the minimum away from zero (which determines the mass of the radial scalar mode) never stops. This is due to the presence of Goldstone modes at this point, which never decouple. In table 2 we summarize the characteristics of the phase transition for the model of fig. 10. The critical temperature and the discontinuity in the order parameter are given in the first three rows. The value of the coarse-graining scale is presented next. The correlation lengths of the gauge field and the radial scalar mode can be inferred from the masses at the two minima, given in the next four rows. The gauge field stays massless at the origin. We have found no evidence that it develops a thermal mass. The last four rows give the values of the surface tension and the latent heat of the phase transition, defined according to

$$
\sigma=\int_{0}^{\phi_{0 R}} d \phi \sqrt{2 U\left(\rho, T_{c r}\right)}
$$

and

$$
\Delta Q=\left.T \frac{\partial}{\partial T} U\left(\rho_{0 R}, T_{c r}\right)\right|_{T=T_{c r}} .
$$

The well-defined and intuitive picture that we presented in the previous paragraphs is not valid for all first-order phase transitions. In fig. 12 we present the effective average potential for the Abelian Higgs model with $\lambda_{R}=0.1, e_{R}^{2}=0.09$ and $T^{2} / \rho_{0 R} \simeq 2.69$. We observe several differences with respect to fig. 10. The discontinuity in the scalar field 
expectation value is about three times smaller for the potential of fig. 12. The phase transition is more weakly first-order than that of fig. 10. The other important difference is that the potential never becomes relatively stable around a certain form. During the later stages of the evolution, its outer part (for scalar field values larger than the location of the minimum) starts approaching a stable profile, due to the decoupling of the massive modes in this region. However, over the same time the non-convex part starts becoming flatter, as configurations interpolating between the two minima are being integrated out [37. The negative curvature at the top of the barrier is expected to behave $\sim-k^{2}$ during this stage [37. This has been verified explicitly through the analytical integration of the evolution equation for the $O(N)$-symmetric scalar theory in the large- $N$ limit [22, 22]. The technical reason for this behaviour can be traced to the presence of a pole at $w=-1$ for the threshold functions $L_{n}^{d}(w)$ [23, 37]. The contributions to the evolution equation coming from the scalar fluctuations involve threshold functions, with arguments which include the terms $u_{k}^{\prime}=U_{k}^{\prime} / k^{2}$ and $u_{k}^{\prime \prime} \tilde{\rho}=U_{k}^{\prime \prime} \rho / k^{2}$. As the pole cannot be crossed, $U_{k}^{\prime}$ and $U_{k}^{\prime \prime}$ must go to zero with $k^{2}$ in the regions where they are negative. This induces the flattening of the non-convex parts. It is difficult to follow this part of the evolution numerically. For this reason we do not display the full approach to convexity in fig. 12. A more thorough presentation, with more detailed figures, will be given elsewhere [57]. It is clear, however, that the well-defined separation between the integration of high-frequency modes and the study of tunnelling or thermal fluctuations above the barrier does not exist for the model of fig. 12. As a result, one cannot obtain a reliable prediction for the nucleation rate by studying only a dominant semiclassical configuration. A small variation of the coarsegraining scale induces large changes in the characteristics of this configuration and the predicted nucleation rate. In fig. 13 we compare various approximations to the potential at the critical temperature, in complete analogy with fig. 11. The prediction from oneloop perturbation theory is that the inclusion of scalar fluctuations (line (b)) increases the strength of the first-order phase transition. This is counter-intuitive and is contradicted by the integration of the evolution equation for the potential (line (c)). The inclusion of the running of the gauge coupling further reduces the strength of the phase transition. Notice that, in figs. 12 and 13, we have stopped the evolution at a scale $k$ where the two minima have equal depth for a certain choice of the temperature. There is considerable arbitrariness in the value of the temperature that can be defined as the critical one, and, therefore, our choice is not unique. For this reason we have not produced a table with the characteristics of the phase transition. Our present understanding of the dynamics of weakly first-order phase transitions does not permit quantitatively precise predictions for their characteristics. For recent related work, see ref. [58].

\section{The evolution equations for the $S U(2)$ Higgs model}

We now turn to the discussion of the $S U(2)$ Higgs model, which displays all the behaviour characteristic of the electroweak phase transition. The inclusion of the $U(1)$ gauge group and fermions introduces only quantitative modifications, without altering the qualitative 
properties of the phase transition. The evolution equation for the effective average potential can be obtained in complete analogy with the intuitive derivation of section 2 . In three dimensions and in terms of the rescaled variables of eqs. (3.1), it reads

$$
\begin{aligned}
\frac{\partial u_{k}}{\partial t}= & -3 u_{k}+\tilde{\rho} u_{k}^{\prime} \\
& -v_{3} L_{0}^{3}\left(u_{k}^{\prime}+2 u_{k}^{\prime \prime} \tilde{\rho}\right)-\left(4 N_{d}-1\right) v_{3} L_{0}^{3}\left(u_{k}^{\prime}\right)-6 v_{3} L_{0}^{3}\left(2 \tilde{e}^{2} \tilde{\rho}\right) .
\end{aligned}
$$

The only differences in comparison with eq. (5.5) concern the group factors multiplying the contributions of the various modes. We consider a model with $N_{d}$ complex doublets, and there are three gauge-group generators. Notice also that we employ a rather unconventional normalization of the gauge coupling and the gauge field mass. The rigorous formulation that leads to the above equation, for a truncated form of the effective average action analogous to eq. (2.7), can be found in refs. [18, 30, 31. We find it more convenient to solve numerically the evolution equation for the derivative of the potential $u_{k}^{\prime}(\tilde{\rho})$. This is completely analogous to eq. (3.4) with $d=3$ and the appropriate group factors multiplying the various contributions. We neglect the logarithmic running of the gauge coupling in four dimensions. The $\beta$-function for the three-dimensional running of the gauge coupling for the pure $S U(2)$ theory, in a truncation that assumes the standard form for the gauge field kinetic term, was derived in ref. [18. The generalization for the $S U(2)$ Higgs model was conjectured in ref. [31. It involves a threshold function, which suppresses the evolution when the scale $k$ becomes smaller than the running mass of the gauge field fluctuations that drive the evolution. This mass is proportional to the Higgs field expectation value. In section 3, we neglected all threshold behaviour in eq. (3.6) for the running of the gauge coupling. However, this behaviour will be important in the context of the electroweak phase transition. The reason is that the $S U(2)$ theory is asymptotically free and the gauge coupling grows in the infrared. Above a critical value for the coupling, a confining regime is expected to arise. The confining regime appears for small Higgs field expectation values, for which the gauge field mass is small and the running of the coupling is not cut off by the threshold function. We use the following expression for the running of the rescaled gauge coupling

$$
\frac{d \tilde{e}^{2}}{d t}=-\tilde{e}^{2}-\frac{4}{3} v_{3} 88 \tilde{e}^{4} l_{N A}^{3} \theta\left(2 \tilde{e}^{2} \tilde{\rho}\right)
$$

with

$$
l_{N A}^{3}=0.677,
$$

and $v_{d}$ given by eq. (3.3). This is the pure $S U(2)$ result with an additional threshold function, which has been approximated by a theta-function. The evolution of $e^{2}$ is stopped as soon as the scale $k$ becomes smaller than the running mass of the gauge field. This results in a $\rho$-dependent running gauge coupling $e^{2}$. There are no fixed points in the evolution of the gauge coupling, according to eq. (9.2). As a result, second-order phase transitions are not expected for the $S U(2)$ Higgs model in the region where this equation is applicable. In eq. (9.2) we have neglected the contribution of scalar field fluctuations. 
Their effect is small and we have preferred to neglect it completely rather than guess the analogue of the constant $l_{N A}^{3}$ in their contribution. The induced error can be estimated through a comparison with the leading-order result from the $\epsilon$-expansion

$$
\frac{d \tilde{e}^{2}}{d t}=-\epsilon \tilde{e}^{2}-\frac{4}{3} v_{4}\left(88-2 N_{d}\right) \tilde{e}^{4}
$$

It is a very small effect, $\sim 2 \%$. Notice that, if the contribution $\sim N_{d}$ in eq. (9.4) and the threshold function in eq. (9.2) are neglected, the two equations are in qualitative agreement. As eq. (9.4) is derived for $\epsilon \rightarrow 0$, the constant $v_{d}$ takes its value at $d=4$. In contrast, eq. (9.2) has been derived directly in three dimensions. As a result, $v_{3}$ appears in it, along with the constant $l_{N A}^{3}$. (For the four-dimensional running one finds $l_{N A}^{4}=1$ [18.)

The non-zero-temperature effects can be taken into account, in complete analogy to the discussion in section 4 . The problem can be reduced to that of integrating the threedimensional evolution equations (9.1), (9.2) for the effective couplings defined in eqs. (5.3), (5.4). (Notice that $e^{2}$ now depends on $k, \rho$ and T.) The evolution starts at the scale $k=T / \theta_{2}$ (we use $\theta_{2}=1$ ) with the initial condition of eq. (6.11) for the gauge coupling. The initial condition for the effective average potential is given by

$$
\begin{aligned}
U_{T / \theta_{2}}^{\prime}(\rho, T)= & \lambda_{R}\left\{\rho-\rho_{0 R}-\left[\left(4 N_{d}+2\right)+12 \frac{e_{R}^{2}}{\lambda_{R}}\right] 2 v_{4} T^{2}\left(\frac{2 \sqrt{\pi}}{\theta_{2}}-\frac{2 \pi^{2}}{3}\right)\right\} \\
& +U_{A^{0}}^{\prime}(\rho, T) .
\end{aligned}
$$

The contribution $U_{A^{0}}^{\prime}(\rho, T)$ results from the integration of the fluctuations of the $A^{0}$ component of the gauge field. This component develops a thermal mass and soon decouples from the evolution, as we have already explained in sections 5 and 6 . For this reason, there are no infrared problems or non-perturbative dynamics associated with it. It is, therefore, a good approximation to use the one-loop perturbative result for the contribution of the $A^{0}$ component

$$
\begin{aligned}
U_{A^{0}}^{\prime}(\rho, T) & =3 \frac{d}{d \rho}\left\{\frac{T^{4}}{2 \pi^{2}} \int_{0}^{\infty} d x x^{2} \ln \left(1-\exp \left[-\sqrt{x^{2}+\frac{m_{L}^{2}}{T^{2}}}\right]\right)\right\} \\
& =3 \frac{d}{d \rho}\left(\frac{1}{24} m_{L}^{2} T^{2}-\frac{1}{12 \pi} m_{L}^{3} T+\ldots\right),
\end{aligned}
$$

with 51

$$
m_{L}^{2}=2 e_{R}^{2} \rho+\frac{2\left(N_{d}+4\right)}{3} e_{R}^{2} T^{2}
$$

the thermally corrected mass.

Similarly to the study of the Abelian Higgs model, we can check the validity of our approximations by comparing with the perturbative predictions. We can integrate the evolution equation for the potential, in analogy to the end of section 6 , neglecting the 
running of couplings and threshold effects. This predicts a critical temperature in agreement with the lowest-order perturbative result [59]

$$
T_{c r}^{2}=\frac{24 \rho_{0 R}}{\left(4 N_{d}+2\right)+18 \frac{e_{R}^{2}}{\lambda_{R}}} .
$$

Corrections to the above expression arise through the inclusion of the running of couplings and threshold effects in the evolution. Another check can be made through the comparison with the full one-loop perturbative contribution coming from the gauge field, if the scalar contributions and the running of the gauge coupling are neglected. As in section 6, we find a small discrepancy, which is due to an imprecise value for the location of the minimum at the beginning of the three-dimensional evolution. This results from the omission of threshold effects in the four-dimensional evolution, and means that our value for the critical temperature cannot be trusted to better than $0.3 \%$. In order to eliminate this particular source of uncertainty, we determine an empirical shift for the initial value of the minimum $\delta \rho_{0}\left(T / \theta_{2}\right)$, through a comparison with the perturbative result. We incorporate this value in the initial condition of eq. (9.5), so that the perturbative prediction is reproduced, when the scalar contributions and the running of the gauge coupling are neglected.

\section{The electroweak phase transition}

In the previous section we summarized the formalism that we need for the study of the phase transitions in the $S U(2)$ Higgs model. We reduced the problem of calculating the temperature-dependent effective potential to that of integrating the evolution equations (9.1) and (9.2), for the effective three-dimensional parameters (effective average potential and running gauge coupling) defined in eqs. (5.3), (5.4). The evolution starts at the scale $k=T / \theta_{2}$ (we use $\theta_{2}=1$ ), with the initial conditions given by eqs. (9.5)-(9.7) and (6.11) in terms of the renormalized parameters of the zero-temperature theory. The temperature-dependent potential is obtained in the limit $k \rightarrow 0$. The initial condition for the potential incorporates the contributions from the part of the evolution that integrates out fluctuations with four-dimensional character. The remaining part is purely threedimensional. Two algorithms for the numerical integration have been presented in detail in ref. [27. They give results which agree at the $0.3 \%$ level. We expect the numerical solution to be an approximation of the solution of the partial differential equation (9.1) with the same level of accuracy.

In fig. 14 we present the evolution of the effective average potential $U_{k}(\rho, T)$ for the $S U(2)$ Higgs model with $N_{d}=1$, as the coarse-graining scale $k$ is lowered. We use the zero-temperature couplings $\lambda_{R}=0.02024, e_{R}^{2}=0.1073$ and choose the temperature $T^{2} / \rho_{0 R} \simeq 0.279$, so as to be close to the phase transition. As we mentioned in the previous section, we neglect the logarithmic running of the four-dimensional couplings that appear in the initial conditions for the three-dimensional running. It is consistent, therefore, to use the tree-level relations for the masses of the Higgs and gauge field, which 
are $m_{H}=35 \mathrm{GeV}$ and $m_{W}=80.6 \mathrm{GeV}$. The corrections to the above values are expected to be small for the $S U(2)$ Higgs model. However, for the full electroweak theory they can be sizeable, due to the large Yukawa coupling of the top quark. In fig. 14 we observe an evolution analogous to that of fig. 10. Initially the potential has only one minimum away from the origin, which receives a renormalization proportional to the running scale $k$ during the evolution. At some point, a new, very shallow minimum appears at the origin. It is induced by the integration of thermal fluctuations, through the generalization of the Coleman-Weinberg mechanism. As a result the phase transitions for this parameter range are expected to be of first order. The evolution slows down at the later stages and the potential converges towards a non-convex profile. The simultaneous running of the gauge coupling $e^{2}(k, \rho, T)$ is depicted in fig. 15. We observe that the gauge coupling increases, because the $S U(2)$ Higgs model is asymptotically free. There are no fixed points in the evolution for any value of $e_{R}^{2}$, which indicates the absence of second-order phase transitions f. The running of the gauge coupling stops earlier for large $\rho$. The reason is the decoupling of the massive gauge field fluctuations when the running scale $k$ becomes smaller than their mass $\sqrt{2 e^{2} \rho}$. At this point, the evolution is automatically stopped by the theta-function in the r.h.s. of eq. (9.2). For values of $\rho$ near the origin the magnitude of the gauge coupling continues to increase. Eventually it reaches a critical value, for which a confining regime is expected to emerge. An estimate for this value is $\alpha=4 \tilde{e}^{2} / 4 \pi=1$. (The reason for the factor of 4 is our unconventional normalization of the gauge coupling.) In figs. 14 and 15, we have stopped the evolution at this scale, which is determined through the expression

$$
\tilde{e}^{2}\left(k_{\text {conf }}, \tilde{\rho}=0\right)=\frac{e^{2}\left(k_{\text {conf }}, \rho=0, T\right) T}{k_{\text {conf }}}=\pi .
$$

The value of $k_{\text {conf }}$ has a very weak dependence on the value of $\tilde{e}^{2}$ for which confinement is assumed to set in. The reason is that the running coupling diverges very fast at this point of the evolution. It becomes infinite at the scale

$$
k_{d i v}=\frac{e_{R}^{2}}{e_{R}^{2} \theta_{2}+\left(\frac{4}{3} v_{3} 88 l_{N A}^{3}\right)^{-1}} T \simeq 0.1 T,
$$

for $e_{R}^{2}=0.1073$ [31, 35. The difference between the scales $k_{\text {conf }}$ and $k_{\text {div }}$ is $\sim 10 \%$, independently of the precise definition of $k_{\text {conf }}$.

The evolution for $k<k_{\text {conf }}$ cannot be described reliably in terms of a parametrization such as that of eq. (2.7) for the effective average action. Instead, one has to use a basis of composite operators, corresponding to the bound states that are expected to form at these scales [39]. The two formulations must be matched at $k=k_{\text {conf }}$. The various bound states are expected to be massive [38], with masses of the order of $k_{\text {conf }}$, and they should

\footnotetext{
5 A second-order phase transition is expected at the point where the first-order phase transitions are replaced by continuous crossovers. This happens for a Higgs field mass larger than the gauge field mass. The description in terms of fundamental fields and eq. (9.2) cease to be valid at this point. A parametrization of the effective average action in terms of composite operators is necessary [39].
} 
soon decouple. The most significant contribution from this last part of the evolution comes from the appearance of condensates associated with the operator $F^{2}$. In ref. [40] it was shown that $F^{2}$ develops a non-zero expectation value in the confining regime, with a reduction of the energy density compared with the state with $F^{2}=0$. This negative contribution affects the potential of fig. 14. The reason is that the strongly-coupled regime appears only near the origin of the potential, where the running of the gauge coupling has not been cut off. We expect, therefore, a negative contribution to the potential for the region around $\rho=0$, where the coupling satisfies eq. (10.1). As we have pointed out, the detailed treatment of this part of the evolution requires extended truncations and a formulation based on composite operators. We do not embark here on this extensive study, which is the subject of future work. Instead we use a cruder approximation for the contribution associated with the $F^{2}$ condensate. On dimensional grounds, we approximate the negative contribution to the potential by

$$
\Delta U=-\left(c k_{\text {conf }}\right)^{3} T .
$$

The constant $c$ is expected to be of order 1 . We consider the values $c=1$ and 0.5 in order to study the effect of this parameter on the characteristics of the phase transition. In fig. 14 we display the form of the potential, if the above contribution with $c=1$ is added to the result of the integration at $k=k_{\text {conf }}$. This term is added to the potential only in the small region around the origin, where the running coupling of fig. 15 reaches the value determined by eq. (10.1). As a result, the shallow minimum at the origin becomes as deep as the minimum at non-zero $\rho$. The resulting potential incorporates the dominant effects from the integration of the fluctuations in the strongly coupled regime for $k<k_{\text {conf }}$. We have not attempted to account for the $\rho$ dependence of the $F^{2}$ condensate beyond the crude step-like behaviour. In ref. [35 this behaviour was smoothed out through the introduction of additional phenomenological parameters. As our analysis cannot provide any hint on the value of these parameters, we have preferred to neglect them completely. This explains the steep rise of the potential of fig. 14 near the origin. Notice, however, that the influence of this crude approximation on the characteristics of the phase transition is rather small. For example, according to eq. (8.3), the latent heat is determined by the temperature dependence of the energy density at the minimum away from the origin. This depends on the magnitude of the contribution of eq. (10.3), but not on its precise $\rho$ dependence. Similarly, the surface tension given by eq. (8.2) involves an integration over the whole range between the two minima, which reduces the effect of our approximation. We conclude that the most significant uncertainty in our treatment of the confining regime is related to the value of the parameter $c$ in eq. (10.3).

The potential at the end of the evolution in fig. 14 has the properties of the nonderivative part of a coarse-grained free enery, as we discussed in detail in section 8 . The convergence towards a non-convex profile, before the strongly-coupled regime is reached, indicates that the various massive modes have already started to decouple. The part of the integration until the decoupling of the bound states is not expected to change this behaviour, as it is rather short (the mass scale for these states is set by $k_{\text {conf }}$ ). As a 
result, we expect the separation in two stages that we observed in section 8 . First, the integration of high-frequency modes generates a non-convex potential with the properties of the non-derivative part of a coarse-grained free energy. In this potential, tunnelling or thermal fluctuations over the barrier can be studied through semiclassical techniques. In fig. 16 we compare various approximations for the form of the potential at the respective critical temperatures of the first-order phase transitions for the model of fig. 14. Line (a) is the perturbative one-loop result. For the scalar fluctuations we have used expressions analogous to eq. (9.6), with thermally corrected masses given by

$$
\begin{aligned}
& m_{G}^{2}=\lambda_{R} \rho+\left(\frac{3}{4} e_{R}^{2}+\frac{2 N_{d}+1}{12} \lambda_{R}\right)\left(T^{2}-\frac{12 \rho_{0 R}}{\left(2 N_{d}+1\right)+9 \frac{e_{R}^{2}}{\lambda_{R}}}\right) \\
& m_{R}^{2}=3 \lambda_{R} \rho+\left(\frac{3}{4} e_{R}^{2}+\frac{2 N_{d}+1}{12} \lambda_{R}\right)\left(T^{2}-\frac{12 \rho_{0 R}}{\left(2 N_{d}+1\right)+9 \frac{e_{R}^{2}}{\lambda_{R}}}\right)
\end{aligned}
$$

for the Goldstone and radial modes respectively. Line (b) results from the integration of the evolution equation if the running of $e^{2}$ is neglected. We observe that the strength of the first-order phase transition is reduced. We have observed the same behaviour in section 8. The proper integration of scalar fluctuations through the renormalization group reduces the size of the barrier, contrary to the predictions of perturbation theory. Line (c) results from the integration of the evolution equation for the potential with the running of $e^{2}$ included, but neglecting the effect of the $F^{2}$ condensate. The presented potential corresponds to the coarse-graining scale $k_{b}$, where the evolution slows down, in complete analogy to the discussion in section 8. As the first-order phase transition is triggered by the gauge field fluctuations, its strength is increased compared with line (b), because the "effective" gauge coupling is larger than $e_{R}^{2}$. Line (d) incorporates the contribution of eq. (10.3) with $c=1$, which comes from the $F^{2}$ condensate. We observe a dramatic increase of the strength of the first-order phase transition. Line (e) is similar to line (d), but for a value $c=0.5$. The influence of the condensate diminishes, but again an increase of the strength of the phase transition is observed. We should also mention that this increase is observed in higher orders of perturbation theory, where the running of the couplings is partly accounted for [6].

The characteristics of the phase transition can be deduced from the form of the potential, in complete analogy to the discussion of section 8. In table 3 we summarize them for the model of fig. 14. The three columns correspond to the curves (c), (d) and (e) of fig. 16. The critical temperature and the discontinuity in the order parameter are given in the first three rows. The value of the scale $k_{\text {conf }}$ where confinement sets in is presented next. We do not give a value for $k_{\text {conf }}$ in the first column, because the effects of the strongly-coupled regime are not taken into account for the potential of line (c). The correlation lengths of the gauge field and the radial scalar mode at the minimum away from the origin can be inferred from the masses, given in the next three rows. We do not give any values for the masses at the origin. There, the proper description should 
include bound states instead of fundamental fields, and is the subject of future work. We have given the value of the mass of the radial scalar mode for two scales. The reason is that the running of the quartic coupling (which determines this mass) never stops. This is due to the presence of Goldstone modes at the minimum away from the origin, which never decouple. In contrast, the running of the gauge coupling (which determines the mass of the gauge field) is stopped by the threshold function in eq. (9.2). For line (e), the confinement scale where we stop the evolution is larger than the mass of the radial mode. The last four rows give the values of the surface tension and the latent heat of the phase transition, defined according to eqs. (8.2) and (8.3), respectively. The influence of the confining regime on the characteristics of the phase transition is apparent. Also, the dependence on the parameter $c$ in eq. (10.3) is significant. We can derive a phenomenologically motivated value for this constant by comparing with the results of other approaches for the quantities of table 3 .

The values of table 3 for the critical temperature and the discontinuity in the Higgs field expectation value are in good agreement with the results of ref. [35], obtained for the same approximations for the quantity of eq. (10.3). A more crucial test is provided by comparison with the results from lattice studies. Unfortunately, the studies of refs. [12, 13] where carried out for different electroweak parameters. In ref. [12 different masses for the Higgs field were used. In ref. [13] the value of the renormalized gauge coupling is different. This results in a different value for the minimum of the potential for the zero-temperature theory than the one we used. The direct comparison is in progress [60]. However, there is an indirect way for an immediate comparison. In ref. [61] the lattice results were compared with the predictions of two-loop perturbation theory. According to figs. 7 and 8 of this reference, good agreement is observed for Higgs field masses in the $20-50 \mathrm{GeV}$ range. This agreement cannot be explained by our analysis. Our fig. 16 indicates that the non-perturbative effects of the strongly-coupled regime are substantial already at $m_{H}=35$ $\mathrm{GeV}$. In spite of that, we can use the perturbative results of ref. [61] as a phenomenological fit to the lattice data. We deduce the values $T_{c r} / m_{H} \simeq 2.76$ and $\Delta Q / T_{c r}^{4} \simeq 0.103$ from figs. 4 and 6 in ref. 61 0. They can be compared with the results presented in table 3. We observe that they are in good agreement with column (d). This agreement is also observed through the direct comparison with the lattice results, for Higgs field masses in the 30-50 GeV range 60]. We conclude that the characteristics of the first-order phase transition are properly reproduced by our analysis, if the non-perturbative contribution from the strongly-coupled regime in the evolution of the potential is approximated by eq. (10.3) with $c=1$. The non-perturbative effects dramatically increase the strength of the phase transition. Their effect is much larger than the effect due to the growth of the running gauge coupling. We should point out, however, that the determination of the value $c=1$ through an explicit calculation in the context of the effective average action is still pending.

\footnotetext{
${ }^{6}$ The quantity $\phi_{0}\left(T_{c r}\right) / T_{c r} \simeq 1.26$, which can be deduced form fig. 5 , corresponds to the expectation value of the operator $\phi^{*} \phi$. The values quoted in our table 3 correspond to expectation values of $\phi$ in a formalism with gauge fixing.
} 
We now turn to the question of the nature of the phase transition for large Higgs field masses. In fig. 17 we plot the effective average potential $U_{k}(\rho, T)$ as the coarse-graining scale $k$ is lowered. The Higgs and gauge field masses are $m_{H}=70 \mathrm{GeV}$ and $m_{W}=80.6$ $\mathrm{GeV}$ respectively, and the temperature $T^{2} / \rho_{0 R} \simeq 0.882$. We observe the running of the minimum and the curvature at the origin becoming less negative. In the region around the origin, the running gauge coupling reaches the critical value of eq. (10.1), at which the confining regime is expected to set in. The size of this region relative to the location of the minimum of the potential is larger than in the case of fig. 14. Confinement sets in while the curvature at the origin is still negative. At this point we stop the evolution and add the contribution of eq. (10.3) to the potential, in the region around the origin where the coupling has the critical value. (We display the evolution with $c=0.5$, but we give the characteristics of the transition for both $c=1$ and 0.5.) This results in a new minimum between the origin and the original minimum of the potential. A first-order phase transition is predicted, but both minima now exist at non-zero values of the Higgs field. This is the major difference between figs. 17 and 14. Other differences concern the strength of the predicted first-order phase transition. The discontinuity in the Higgs field expectation value is smaller in fig. 17. Also the potential does not converge towards a stable profile, which would indicate the decoupling of massive modes. This is the typical behaviour of weakly first-order phase transitions, which we have already discussed in detail in section 8. As we have mentioned at the end of that section, the dynamics of such phase transitions is not well understood. In table 4, we list the characteristics which are predicted by the potential at the end of the evolution in fig. 17, in complete analogy to table 3. The location of the new minimum is also included in the table. The latent heat is calculated through

$$
\Delta Q=T \frac{\partial}{\partial T}\left\{U\left(\rho_{0 R}, T_{c r}\right)-U\left(\rho_{n p}, T_{c r}\right)\right\}_{T=T_{c r}} .
$$

We point out that, similarly to section 8 , there is considerable arbitrariness in defining which temperature is the critical one, and, therefore, the values of table 4 must be viewed as indicative, rather than quantitatively precise. The appearance of a minimum at a non-zero Higgs expectation value has also been observed in ref. [35] and within the gapequation approach in ref. [9].

For even larger Higgs masses a qualitative change is expected. The evolution resembles that in fig. 17, until the gauge coupling reaches its critical value. When the stronglycoupled regime sets in, its extent is larger than the location of the minimum of the potential. This indicates that there is no longer any minimum where the description in terms of fundamental fields is possible. The transition to a non-perturbative vacuum is expected to be continuous, without the appearance of singularities. As a result there is no phase transition any more. This indicates the change from first-order phase transitions to analytical crossovers for large Higgs field masses. Our analysis predicts a critical Higgs field mass in the range $80-100 \mathrm{GeV}$. The possibility of a crossover was suggested in ref. 31]. It is supported by the studies of ref. [35], where arguments similar to ours were given, and ref. [9], where the gap-equation approach was followed. The most reliable 
results that confirm this possibility have been obtained through the lattice approach [62].

\section{Conclusions}

In this paper we discussed the high-temperature phase transitions for the Abelian and $S U(2)$ Higgs models by employing the method of the effective average action. Our aim was to present a method that can deal with the most important problems to be encountered in the study of the electroweak phase transition. We identified as such the problem of infrared divergences in the perturbative approach and the absence of a coarse-graining scale in the calculation of potentials, which are used for the study of first-order phase transitions. The method of the effective average action provides a resolution of these problems through the introduction of an infrared cutoff scale $k$. This can be identified with the coarse-graining scale. The effective average potential has the characteristics of the non-derivative part of a coarse-grained free energy, on which a proper treatment of statistical systems can be based. It does not have to be convex and it is the most natural tool for the study of first-order phase transitions. The resolution of the problem of infrared divergences is achieved through the use of the renormalization group, which is built in the method. The dependence of the effective average action on the scale $k$ is described by an exact renormalization-group equation. From this, evolution equations for the potential and other invariants can be derived. The integration of the evolution equations determines all the couplings of the renormalized theory at zero and non-zero temperature without the appearance of any non-physical divergences. The reduction of the effective dimensionality of the system from four to three, at scales $k$ smaller than the temperature, is easily demonstrated. The complete phase diagram can be determined without the need to resort to lattice simulations, which require long computer time and do not provide intuition on the nature of the physical behaviour. Moreover, the discussion of the phase diagram in terms of the evolution of the potential provides information on the running of all the generalized couplings of the theory (the 1PI Green's functions at zero external momenta). It also gives a detailed picture of first-order phase transitions, for which the shape of the whole potential, and not the running of a few couplings, is required. The third important problem in the study of the electroweak phase transition concerns the strongly-coupled regime in the symmetric phase of the electroweak theory. The possibility for a proper treatment is provided within this approach, through the use of composite operators for the parametrization of the effective average action in this regime. We did not embark here on this extensive study, which is the subject of future work. Instead we relied on a cruder determination of the contribution to the potential coming from the effects of the confining regime. Through the integration of the evolution equations we determined the potential at the scale $k_{\text {conf }}$, where confinement is expected to set in and an $F^{2}$ condensate appears. The effect on the potential was determined from this scale through a dimensional argument, which introduces an undetermined parameter. This was fixed through the comparison of our results with those of the lattice approach.

We found that the Abelian Higgs model with a number of complex scalar fields $N_{c} \geq 5$ 
has a region of second-order phase transitions and a region of first-order ones, which are separated by a tricritical point. The second-order phase transitions are governed by two fixed points: the Wilson-Fisher fixed point and the Abelian one. We determined the universal form of the potential at the fixed points and the tricritical one, from which the values of all the generalized couplings can be obtained (by taking derivatives with respect to the field). Moreover, we investigated through the evolution of the potential the relative stability of the fixed points. We determined critical exponents and crossover curves, which parametrize the physical behaviour near the critical temperature. For $N_{c}<5$ the fixed points disappear and only first-order phase transitions exist. Our determination of the critical $N_{c}$ for the qualitative change of behaviour is subject to uncertainties, due to the truncated form of the effective average action that we used. We can firmly establish that $\left(N_{c}\right)_{c r}=\mathcal{O}(1)$. However, the existence of the region of secondorder phase transitions for $N_{c}=1$ is not excluded. This leaves open the possibility of a second-order phase transition for low-temperature superconductors, which belong to the same universality class as the Abelian Higgs model. The first-order phase transitions were studied in detail. We presented an explicit realization of a coarse-grained potential for a strongly first-order phase transition, from which its characteristics were derived. We showed that the study of such phase transitions can be naturally separated in two parts: First the high-frequency modes are integrated out and a non-convex potential is generated (often through the Coleman-Weinberg mechanism of radiative symmetry breaking). At a second stage, the fluctuations that drive the phase transition (instantons or critical bubbles) can be studied with semiclassical techniques, using the potential. This separation is impossible for weakly first-order phase transitions. The two parts merge, and the dominant semiclassical configurations do not provide a complete description.

The $S U(2)$ Higgs model with one scalar doublet (which exhibits the same qualitative behaviour as the electroweak theory) has only first-order phase transitions for Higgs field masses smaller than the gauge field mass. The gauge coupling near the origin of the potential grows as $k$ is lowered, until it reaches a critical value, for which a confining regime is expected to set in. At this point an $F^{2}$ condensate emerges with a negative contribution to the potential around the origin. This increases dramatically the strength of the first-order phase transition. We determined its characteristics for $m_{H}=35 \mathrm{GeV}$, which are in good agreement with two-loop perturbation theory and lattice results. We also considered the case $m_{H}=70 \mathrm{GeV}$, for which the first-order phase transition is much weaker. The behaviour that we described above in the context of the Abelian Higgs model for such transitions was again observed. For Higgs masses above $80-100 \mathrm{GeV}$ we no longer found a two-minimum potential with a prediction of a first-order transition. Instead we found indications that an analytical crossover connects the two regions of the phase diagram that correspond to the symmetric phase and the phase with spontaneous symmetry breaking.

The picture that emerged from our study of the high-temperature phase transitions for the Abelian and $S U(2)$ Higgs models has a rich structure and provides physical intuition as well as quantitative information. The method of the effective average action has 
also been applied to pure scalar theories (a list of references is given in the introduction). The second-order phase transitions of the $O(N)$-symmetric theory have been discussed in terms of the evolution of the potential, with the identification of fixed points. Critical exponents and amplitudes, as well as the full critical equation of state, have been computed. Two-scalar theories have also been considered, which exhibit a richer fixed-point structure, crossover phenomena and first-order phase transitions. The combined picture of phase transitions for high temperature field theories incorporates a wide range of physical behaviours. The most important pending problem in this approach is the use of a truncated ansatz for the effective average action. An intrinsic check of the accuracy of the predictions can be achieved through the calculation of the change that a more general ansatz induces to the results. A systematic way of carrying out this check has not been firmly established yet. This is the most important direction for further work, which will lead to improved quantitative accuracy.

Acknowledgements: We would like to thank Z. Fodor, M. Shaposhnikov and C. Wetterich for discussions. 


\section{References}

[1] D.A. Kirzhnits and A.D. Linde, Phys. Lett. B 42, 471 (1972).

[2] V.A. Kuzmin, V.A. Rubakov, and M.E. Shaposhnikov, Phys. Lett. B 155, 36 (1985); M.E. Shaposhnikov, Nucl. Phys. B 287, 757 (1987); ibid. 299, 797 (1988).

[3] V.A. Rubakov and M.E. Shaposhnikov, preprint CERN-TH/96-13, hep-ph/9603208.

[4] K. Jansen, preprint DESY-95-169, hep-lat/9509018.

[5] A.I. Bochkarev and M.E. Shaposhnikov, Mod. Phys. Lett. A 2, 417 (1987). G.W. Anderson and L. Hall, Phys. Rev. D 45, 2685 (1992); M. Dine, R. Leigh, P. Huet, A. Linde and D. Linde, Phys. Rev. D 46, 550 (1992); Phys. Lett. B 283, 319 (1992); M. Carrington, Phys. Rev. D 45, 2933 (1992); M.E. Shaposhnikov, Phys. Lett. B 277, 324 (1992).

[6] J.E. Bagnasco and M. Dine, Phys. Lett. B 303, 308 (1993); P. Arnold and O. Espinosa, Phys. Rev. D 47, 3546 (1993); Z. Fodor and A. Hebecker, Nucl. Phys. B 432, 127 (1994); K. Farakos, K. Kajantie, K. Rummukainen and M. Shaposhnikov, Nucl. Phys. B 425, 67 (1994); M. Laine, Phys. Lett. B 335, 173 (1994); Phys. Rev. D 51, 4525 (1995); J. Kripfganz, A. Laser and M.G. Schmidt, Phys. Lett. B 351, 266 (1995).

[7] A.D. Linde, Phys. Lett. B 96, 293 (1980); D. Gross, R. Pisarski and L. Yaffe, Rev. Mod. Phys. 53, 43 (1981).

[8] W. Buchmüller, Z. Fodor, T. Helbig and D. Walliser, Ann. Phys. 234, 260 (1994).

[9] W. Buchmüller and O. Philipsen, Phys. Lett. B 354, 403 (1995); Nucl. Phys. B 443, 47 (1995).

[10] P. Arnold and G. Yaffe, Phys. Rev. D 49, 3003 (1994).

[11] B. Bunk, E.-M. Ilgenfritz, J. Kripfganz and A. Schiller, Nucl. Phys. B 403, 453 (1993); M. Gürtler, E.-M. Ilgenfritz, J. Kripfganz, H. Perlt and A. Schiller, preprint UL-NTZ-23/96, hep-lat/9605042.

[12] K. Kajantie, K. Rummukainen and M. Shaposhnikov, Nucl. Phys. B 407, 356 (1993); K. Farakos, K. Kajantie, K. Rummukainen and M. Shaposhnikov, Phys. Lett. B 336, 494 (1994); Nucl. Phys. B 442, 317 (1995); K. Kajantie, M. Laine, K. Rummukainen and M. Shaposhnikov, Nucl. Phys. B 466, 189 (1996).

[13] Z. Fodor, J. Hein, K. Jansen, A. Jaster, I. Montvay and F. Csikor, Phys. Lett. B 334, 405 (1994); F. Csikor, Z. Fodor, J. Hein and J. Heitger, Phys. Lett. B 357, 156 (1995); Z. Fodor, J. Hein, K. Jansen, A. Jaster and I. Montvay, Nucl. Phys. B 439, 147 (1995); F. Csikor, Z. Fodor, J. Hein, A. Jaster and I. Montvay, preprint DESY-95-206, hep-lat/9601016. 
[14] K.G. Wilson and I.G. Kogut, Phys. Rep. 12, 75 (1974).

[15] C. Wetterich, Nucl. Phys. B 352, 529 (1991); Z. Phys. C 57, 451 (1993).

[16] L.P. Kadanoff, Physics 2, 263 (1966).

[17] C. Wetterich, Phys. Lett. B 301, 90 (1993).

[18] M. Reuter and C. Wetterich, Nucl. Phys. B 417, 181 (1994).

[19] F.J. Wegner and A. Houghton, Phys. Rev. A 8, 401 (1973); F.J. Wegner, in: Phase transitions and critical phenomena, vol. 6, eds. C. Domb and M.S. Green (Academic Press, New York, 1976); S. Weinberg, Critical phenomena for field theorists, in Erice Subnuc. Phys. 1 (1976); J. Polchinski, Nucl. Phys. B 231, 269 (1984); A. Hasenfratz and P. Hasenfratz, Nucl. Phys. B 270, 687 (1986); J.F. Nicoll and T.S. Chang, Phys. Lett. A 62, 287 (1977); T.S. Chang, J.F. Nicoll and J.E. Young, Phys. Lett. A 67, 287 (1978); U. Ellwanger, Phys. Lett. B 335, 364 (1994).

[20] M. Bonini, M. D'Attanasio and G. Marchesini, Nucl. Phys. B 418, 81 (1994); ibid. 421, 429 (1994); ibid. 437, 163 (1995); ibid. 444, 602 (1995); M. D'Attanasio and T.R. Morris, preprint SHEP-96-08, hep-th/9602156.

[21] N. Tetradis and C. Wetterich, Nucl. Phys. B 398, 659 (1993); Int. J. Mod. Phys. A 9, 4029 (1994).

[22] M. Reuter, N. Tetradis and C. Wetterich, Nucl. Phys. B 401, 567 (1993).

[23] N. Tetradis and C. Wetterich, Nucl. Phys. B 422, 541 (1994).

[24] S. Bornholdt, N. Tetradis and C. Wetterich, Phys. Rev. D 53, 4552 (1996).

[25] S. Bornholdt, P. Büttner, N. Tetradis and C. Wetterich, preprint CERN-TH/96-67, cond-mat/9603129.

[26] S. Bornholdt, N. Tetradis and C. Wetterich, Phys. Lett. B 348, 89 (1995).

[27] J. Adams, J. Berges, S. Bornholdt, F. Freire, N. Tetradis and C. Wetterich, Mod. Phys. Lett. A 10, 2367 (1995).

[28] J. Berges, N. Tetradis and C. Wetterich, preprint OUTP-95-27P, hep-th/9507159.

[29] N. Tetradis and D. Litim, Nucl. Phys. B 464, 492 (1996).

[30] M. Reuter and C. Wetterich, Nucl. Phys. B 391, 147 (1993); ibid. 427, 291 (1994).

[31] M. Reuter and C. Wetterich, Nucl. Phys. B 408, 91 (1993).

[32] D. Litim, C. Wetterich and N. Tetradis, preprint HD-THEP-94-23, hep-ph/9407267. 
[33] S. Coleman and E. Weinberg, Phys. Rev. D 7, 1888 (1973).

[34] B. Bergerhoff, D. Litim, S. Lola and C. Wetterich, preprint HD-THEP-94-49, condmat/9502039; B. Bergerhoff, F. Freire, D. Litim, S. Lola and C. Wetterich, Phys. Rev. B 53, 5734 (1996).

[35] B. Bergerhoff and C. Wetterich, Nucl. Phys. B 440, 171 (1995); preprint HD-THEP95-37, hep-ph/9508352.

[36] T. Papenbrock and C. Wetterich, Z. Phys. C 65, 519 (1995); T.R. Morris, Nucl. Phys. B 458, 477 (1996); C. Wetterich, preprint HD-THEP-96-2, hep-th/9602039.

[37] A. Ringwald and C. Wetterich, Nucl. Phys. B 334, 506 (1990); N. Tetradis and C. Wetterich, Nucl. Phys. B 383, 197 (1992).

[38] E.-M. Ilgenfritz, J. Kripfganz, H. Perlt and A. Schiller, Phys. Lett. B 356, 561 (1995); O. Philipsen, M. Teper and H. Wittig, preprint OUTP-95-40P, hep-lat/9602006; F. Karsch, T. Neuhaus, A. Patkós and J. Rank, preprint BI-TP-96/10, hep-lat/9603004.

[39] U. Ellwanger, Z. Phys. C 58, 619 (1993); U. Ellwanger and C. Wetterich, Nucl. Phys. B 423, 137 (1994).

[40] M. Reuter and C. Wetterich, preprint HD-THEP-94-39, hep-th/9411227.

[41] F. Freire and C. Wetterich, preprint HD-THEP-96-01, hep-th/9601081.

[42] U. Ellwanger, M. Hirsch and A. Weber, Z. Phys. C 69, 687 (1996); preprint LPTHEORSAY-96-50, hep-ph/9606468.

[43] P. Ginsparg, Nucl. Phys. B 170, 388 (1980).

[44] J. March-Russell, Phys. Lett. B 296, 364 (1992).

[45] J. Kapusta, Finite temperature field theory, (Cambridge University Press, 1989).

[46] L. Dolan and R. Jackiw, Phys. Rev. D 9, 3320 (1974).

[47] S. Weinberg, Phys. Rev. D 9, 3357 (1974).

[48] D.A. Kirzhnits and A.D. Linde, JETP 40, 628 (1974); Ann. Phys. 101, 195 (1976).

[49] W. Buchmüller, T. Helbig and D. Walliser, Nucl. Phys. B 407, 387 (1993).

[50] A. Hebecker, Z. Phys. C 60, 271 (1993).

[51] F. Karsch, T. Neuhaus and A. Patkós, Nucl. Phys. B 441, 629 (1995); K. Kajantie, M. Laine, K. Rummukainen and M. Shaposhnikov, Nucl. Phys. B 458, 90 (1996).

[52] D.J. Amit, Field theory, the renormalization group, and critical phenomena, (World Scientific, Singapore, 1984). 
[53] C. Dasgupta and B. I. Halperin, Phys. Rev. Lett. 47, 1556 (1981); J. Bartholomew, Phys. Rev. B 28, 5378 (1983); H. Kleinert, Lett. Nuovo Cim. 35, 405 (1982); J. March-Russell, Phys. Lett. B 296, 364 (1992); M. Kiometzis, H. Kleinert and A.M.J. Schakel, Phys. Rev. Lett. 73, 1975 (1994); L. Radzihovsky, Europhys. Lett. 29, 227 (1995).

[54] J. Langer, Ann. Phys. 41, 108 (1967); ibid. 54, 258 (1969); Physica 73, 61 (1974).

[55] A. D. Linde, Nucl. Phys. B 216, 421 (1983).

[56] K. Takahashi, Z. Phys. C 26, 601 (1985); J.R. Espinosa, M. Quiros and F. Zwirner, Phys. Lett. B 291, 115 (1992).

[57] N. Tetradis and C. Wetterich, in preparation.

[58] M. Gleiser and E.W. Kolb, Phys. Rev. Lett. 69, 1304 (1992); Phys. Rev. D 48, 1560 (1993); N. Tetradis, Z. Phys. C 57, 331 (1993); G. Gelmini and M. Gleiser, Nucl. Phys. B 419, 129 (1994); M. Gleiser, Phys. Rev. Lett. 73, 3495 (1994).

[59] M. Sher, Phys. Rep. 179, 273 (1989).

[60] N. Tetradis, in preparation.

[61] W. Buchmüller, Z. Fodor and A. Hebecker, Nucl. Phys. B 447, 317 (1995).

[62] K. Kajantie, M. Laine, K. Rummukainen and M. Shaposhnikov, preprint CERN$\mathrm{TH} / 96-126$, hep-ph/9605288. 


\section{Figures}

Fig. 1 The effective potential for the Abelian Higgs model with $N_{c}=1$ in the approximation that scalar fluctuations are neglected. $\lambda_{R}=0.02, e_{R}^{2}=0.09, T^{2} / \rho_{0 R} \simeq 0.953$. Line (a) is the perturbative one-loop result.

Line (b) is the result of the numerical integration of the evolution equation without the scalar contributions.

Line (c) is the result of the numerical integration if the initial value for the minimum of the potential is shifted by a relative amount $\simeq 0.3 \%$.

Fig. 2 The phase diagram for the Abelian Higgs model with $N_{c}=250$. The evolution equations (5.6), (3.9) with $d=3$ are used. The critical value for the existence of three fixed points is $\left(N_{c}\right)_{c r}=222$.

Correction: $\tilde{\lambda}, \tilde{e}^{2}$ instead of $\lambda, e^{2}$. The direction of flows is described in the text.

Fig. 3 The running $u^{\prime}(\tilde{\rho})$ for the Abelian Higgs model with $N_{c}=5 . \quad \lambda_{R}=0.5, e_{R}^{2}=$ $10^{-6} \times e_{A}^{2}, T^{2} / \rho_{0 R} \simeq 2.10$. The system approaches first the Wilson-Fisher fixed point and subsequently the Abelian fixed point. The final running leads to the phase with spontaneous symmetry breaking.

Correction: $\tilde{\rho}$ instead of $\rho$.

Fig. 4 The evolution of $\kappa, \lambda$ and $e^{2}$ for the model of fig. 3. The approach to the two fixed points is apparent.

Correction: $\tilde{\lambda}, \tilde{e}^{2}$ instead of $\lambda, e^{2}$.

Fig. 5 The "effective" exponent $\nu$ as the critical temperature is approached for the model of fig. 3 .

Fig. 6 The Wilson-Fisher (WF), Abelian (A) and tricritical (T) fixed point, and the inflection point (I), for $N_{c}=5$.

Correction: $\tilde{\rho}$ instead of $\rho$.

Fig. 7 The phase diagram for the Abelian Higgs model with $N_{c}=1$. The evolution equations (5.6), (3.9) with $d=3$ are used.

Correction: $\tilde{\lambda}, \tilde{e}^{2}$ instead of $\lambda, e^{2}$. The direction of flows is described in the text. 
Fig. 8 The running $u^{\prime}(\tilde{\rho})$ for the Abelian Higgs model with $N_{c}=1 . \quad \lambda_{R}=0.5, e_{R}^{2}=$ $10^{-7} \times e_{A}^{2}, T^{2} / \rho_{0 R} \simeq 6.41$. The system first approaches the Wilson-Fisher fixed point. Subsequently a new minimum appears at the origin, which eventually becomes the absolute minimum of the potential.

Correction: $\tilde{\rho}$ instead of $\rho$.

Fig. 9 The evolution of $\kappa, \lambda$ and $e^{2}$ for the model of fig. 8 .

Correction: $\tilde{\lambda}, \tilde{e}^{2}$ instead of $\lambda, e^{2}$.

Fig. 10 The effective average potential $U_{k}(\rho, T)$ for the Abelian Higgs model with $N_{c}=1$ as the coarse-graining scale $k$ is lowered. $\lambda_{R}=0.02, e_{R}^{2}=0.09, T^{2} / \rho_{0 R} \simeq 0.841$.

Fig. 11 The potential at the critical temperature in various approximations for the model of fig. 10 .

Line (a) is the perturbative one-loop result if the scalar fluctuations are neglected. Line (b) is the perturbative one-loop result with the scalar fluctuations included.

Line (c) results from the integration of the evolution equation if the running of $e^{2}$ is neglected.

Line (d) results from the integration of the evolution equation with the running of $e^{2}$ included.

Fig. 12 Same as fig. 10 for $\lambda_{R}=0.1, e_{R}^{2}=0.09, T^{2} / \rho_{0 R} \simeq 2.69$.

Fig. 13 Same as fig. 11 for the model of fig. 12 .

Fig. 14 The effective average potential $U_{k}(\rho, T)$ for the $S U(2)$ Higgs model with $N_{d}=1$ as the coarse-graining scale $k$ is lowered. The couplings correspond to $m_{H}=35 \mathrm{GeV}$, $m_{W}=80.6 \mathrm{GeV}\left(\lambda_{R}=0.02024, e_{R}^{2}=0.1073\right) . T^{2} / \rho_{0 R} \simeq 0.279$.

Fig. 15 The running gauge coupling $e^{2}(k, \rho, T)$ for the model of fig. 14 .

Fig. 16 The potential at the critical temperature in various approximations for the model of fig. 14 .

Line (a) is the perturbative one-loop result.

Line (b) results from the integration of the evolution equation if the running of $e^{2}$ is neglected.

Line (c) results from the integration of the evolution equation if the running of $e^{2}$ is included, but the effect of the $F^{2}$ condensate is neglected. 
Lines (d) and (e) result from the integration of the evolution equation if both the running of $e^{2}$ and the effect of the $F^{2}$ condensate (in two different approximations) are included.

Fig. 17 The effective average potential $U_{k}(\rho, T)$ for the $S U(2)$ Higgs model with $N_{d}=1$ as the coarse-graining scale $k$ is lowered. $m_{H}=70 \mathrm{GeV}, m_{W}=80.6 \mathrm{GeV}\left(\lambda_{R}=\right.$ $\left.0.08097, e_{R}^{2}=0.1073\right) . T^{2} / \rho_{0 R} \simeq 0.882$. 


\section{Tables}

\begin{tabular}{|c||c|c|c|c|c|}
\hline$N_{c}$ & 100 & 10 & 8 & 6 & 5 \\
\hline$\nu_{A}$ & 0.989 & 0.894 & 0.865 & 0.810 & 0.759 \\
\hline
\end{tabular}

Table 1: The critical exponent $\nu_{A}$ for the Abelian fixed point and various values of $N_{c}$.

\begin{tabular}{|c|c|}
\hline$T_{c r} / \sqrt{\rho_{0 R}}$ & 0.917 \\
\hline$\phi_{0 R}\left(T_{c r}\right) / \sqrt{\rho_{0 R}}$ & 0.235 \\
\hline$\Delta \phi\left(T_{c r}\right) / T_{c r}$ & 0.256 \\
\hline$k_{b} / \sqrt{\rho_{0 R}}$ & $0.985 \times 10^{-2}$ \\
\hline$m_{W}\left(0, T_{c r}\right) / \sqrt{\rho_{0 R}}$ & 0 \\
\hline$m_{W}\left(\rho_{0 R}, T_{c r}\right) / \sqrt{\rho_{0 R}}$ & $0.698 \times 10^{-1}$ \\
\hline$m_{H}\left(0, T_{c r}\right) / \sqrt{\rho_{0 R}}$ & $0.143 \times 10^{-1}$ \\
\hline$m_{H}\left(\rho_{0 R}, T_{c r}\right) / \sqrt{\rho_{0 R}}$ & $0.146 \times 10^{-1}$ \\
\hline$\sigma / \rho_{0 R}^{3 / 2}$ & $0.129 \times 10^{-3}$ \\
\hline$\sigma / T_{c r}^{3}$ & $0.167 \times 10^{-3}$ \\
\hline$\Delta Q / \rho_{0 R}^{2}$ & $0.112 \times 10^{-2}$ \\
\hline$\Delta Q / T_{c r}^{4}$ & $0.158 \times 10^{-2}$ \\
\hline
\end{tabular}

Table 2: Characteristics of the first-order phase transition for the Abelian Higgs model with $N_{c}=1, \lambda_{R}=0.02, e_{R}^{2}=0.09$. 


\begin{tabular}{|c||c|c|c|}
\hline & $(\mathrm{c})$ & $(\mathrm{d})$ & $(\mathrm{e})$ \\
\hline \hline$T_{c r} / \mathrm{GeV}$ & 93.7 & 91.8 & 93.5 \\
\hline$\phi_{0}\left(T_{c r}\right) / \mathrm{GeV}$ & 92.4 & 116 & 96.5 \\
\hline$\Delta \phi\left(T_{c r}\right) / T_{c r}$ & 0.986 & 1.27 & 1.03 \\
\hline$k_{\text {conf }} / \mathrm{GeV}$ & - & 11.7 & 11.9 \\
\hline$m_{W}\left(\rho_{0 R}, T_{c r}\right) / \mathrm{GeV}$ & 33.9 & 41.0 & 35.1 \\
\hline$m_{H}\left(\rho_{0}, T_{c r}, k=m_{H}\right) / \mathrm{GeV}$ & 7.83 & 12.4 & - \\
\hline$m_{H}\left(\rho_{0}, T_{c r}, k=k_{\text {conf }}\right) / \mathrm{GeV}$ & - & 12.3 & 8.68 \\
\hline$\sigma /(\mathrm{GeV})^{3}$ & $1.27 \times 10^{4}$ & $3.04 \times 10^{4}$ & $1.41 \times 10^{4}$ \\
\hline$\sigma / T_{c r}^{3}$ & $1.54 \times 10^{-2}$ & $3.93 \times 10^{-2}$ & $1.73 \times 10^{-2}$ \\
\hline$\Delta Q /(\mathrm{GeV})^{4}$ & $5.46 \times 10^{6}$ & $8.21 \times 10^{6}$ & $5.81 \times 10^{6}$ \\
\hline$\Delta Q / T_{c r}^{4}$ & $7.08 \times 10^{-2}$ & $1.16 \times 10^{-1}$ & $7.60 \times 10^{-2}$ \\
\hline
\end{tabular}

Table 3: Characteristics of the first-order phase transition for the $S U(2)$ Higgs model with $N_{d}=1, m_{H}=35 \mathrm{GeV}, m_{W}=80.6 \mathrm{GeV}$. 


\begin{tabular}{|c||c|c|c|}
\hline & $(\mathrm{c})$ & $(\mathrm{d})$ & $(\mathrm{e})$ \\
\hline \hline$T_{c r} / \mathrm{GeV}$ & 165 & 156 & 163 \\
\hline$\phi_{0}\left(T_{c r}\right) / \mathrm{GeV}$ & 68.0 & 123 & 88.3 \\
\hline$\phi_{n p}\left(T_{c r}\right) / \mathrm{GeV}$ & 0 & 32.1 & 38.1 \\
\hline$\Delta \phi\left(T_{c r}\right) / T_{c r}$ & 0.412 & 0.583 & 0.308 \\
\hline$k_{\text {conf }} / \mathrm{GeV}$ & - & 19.9 & 20.8 \\
\hline$m_{W}\left(\rho_{0 R}, T_{c r}\right) / \mathrm{GeV}$ & 30.8 & 46.4 & 36.6 \\
\hline$m_{H}\left(\rho_{0}, T_{c r}, k=m_{H}\right) / \mathrm{GeV}$ & 8.70 & 29.8 & - \\
\hline$m_{H}\left(\rho_{0}, T_{c r}, k=k_{\text {conf }}\right) / \mathrm{GeV}$ & - & 29.6 & 18.8 \\
\hline$\sigma /(\mathrm{GeV})^{3}$ & $1.03 \times 10^{4}$ & $7.89 \times 10^{4}$ & $1.54 \times 10^{4}$ \\
\hline$\sigma / T_{c r}^{3}$ & $2.29 \times 10^{-3}$ & $2.08 \times 10^{-2}$ & $3.56 \times 10^{-3}$ \\
\hline$\Delta Q /(\mathrm{GeV})^{4}$ & $1.13 \times 10^{7}$ & $3.02 \times 10^{7}$ & $1.56 \times 10^{7}$ \\
\hline$\Delta Q / T_{c r}^{4}$ & $1.52 \times 10^{-2}$ & $5.10 \times 10^{-2}$ & $2.21 \times 10^{-2}$ \\
\hline
\end{tabular}

Table 4: Characteristics of the first-order phase transition for the $S U(2)$ Higgs model with $N_{d}=1, m_{H}=70 \mathrm{GeV}, m_{W}=80.6 \mathrm{GeV}$. 


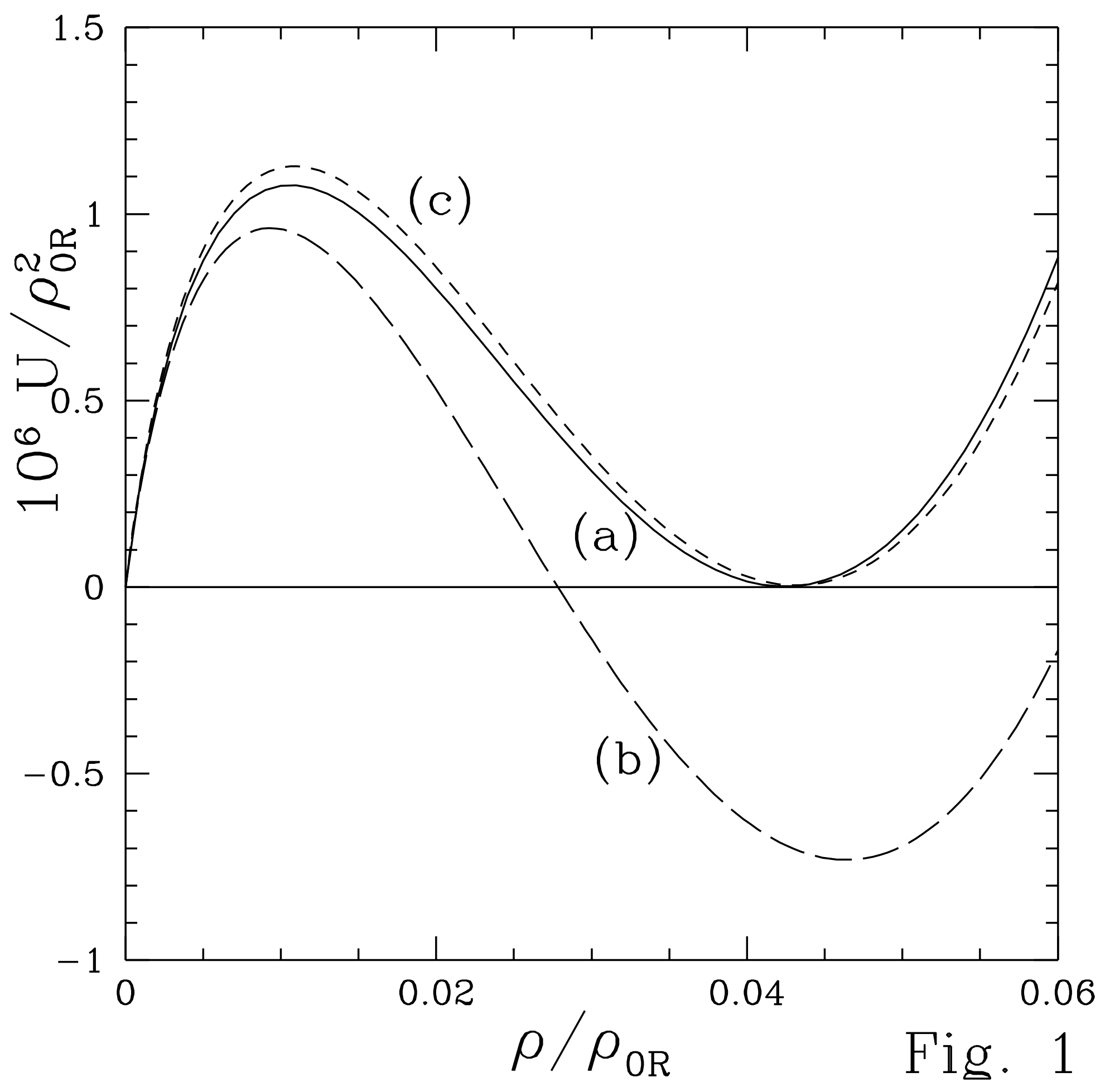




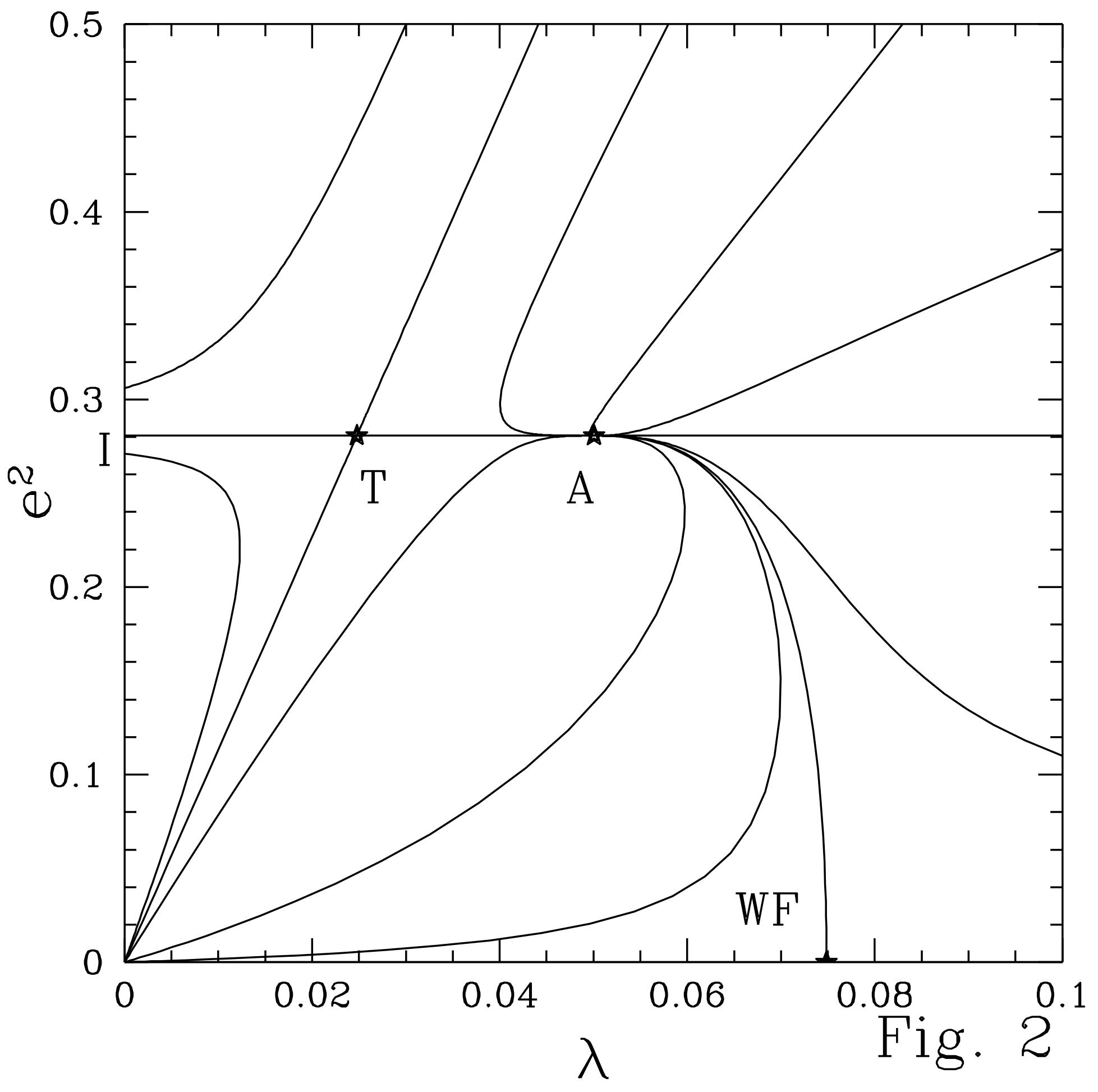




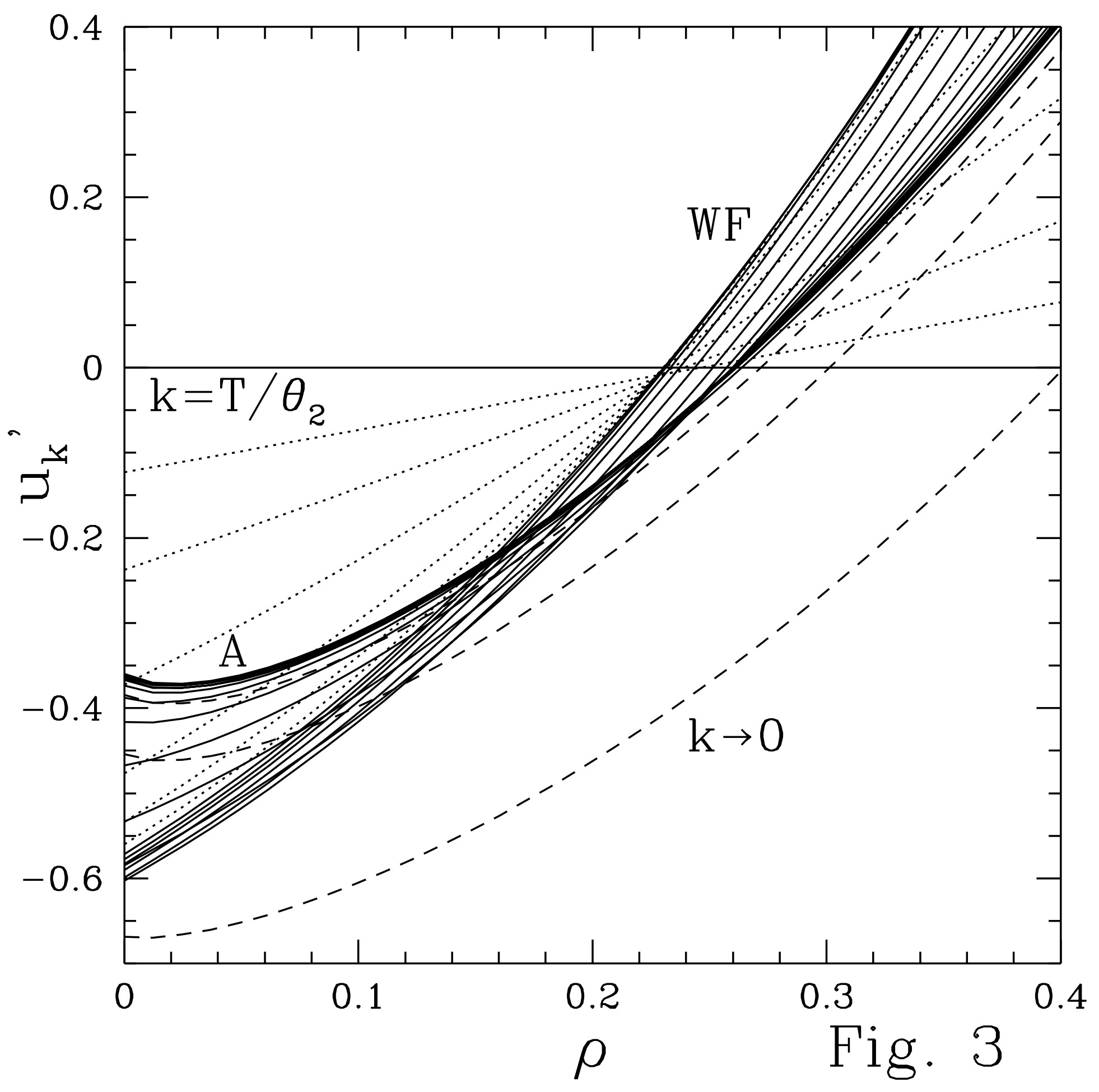




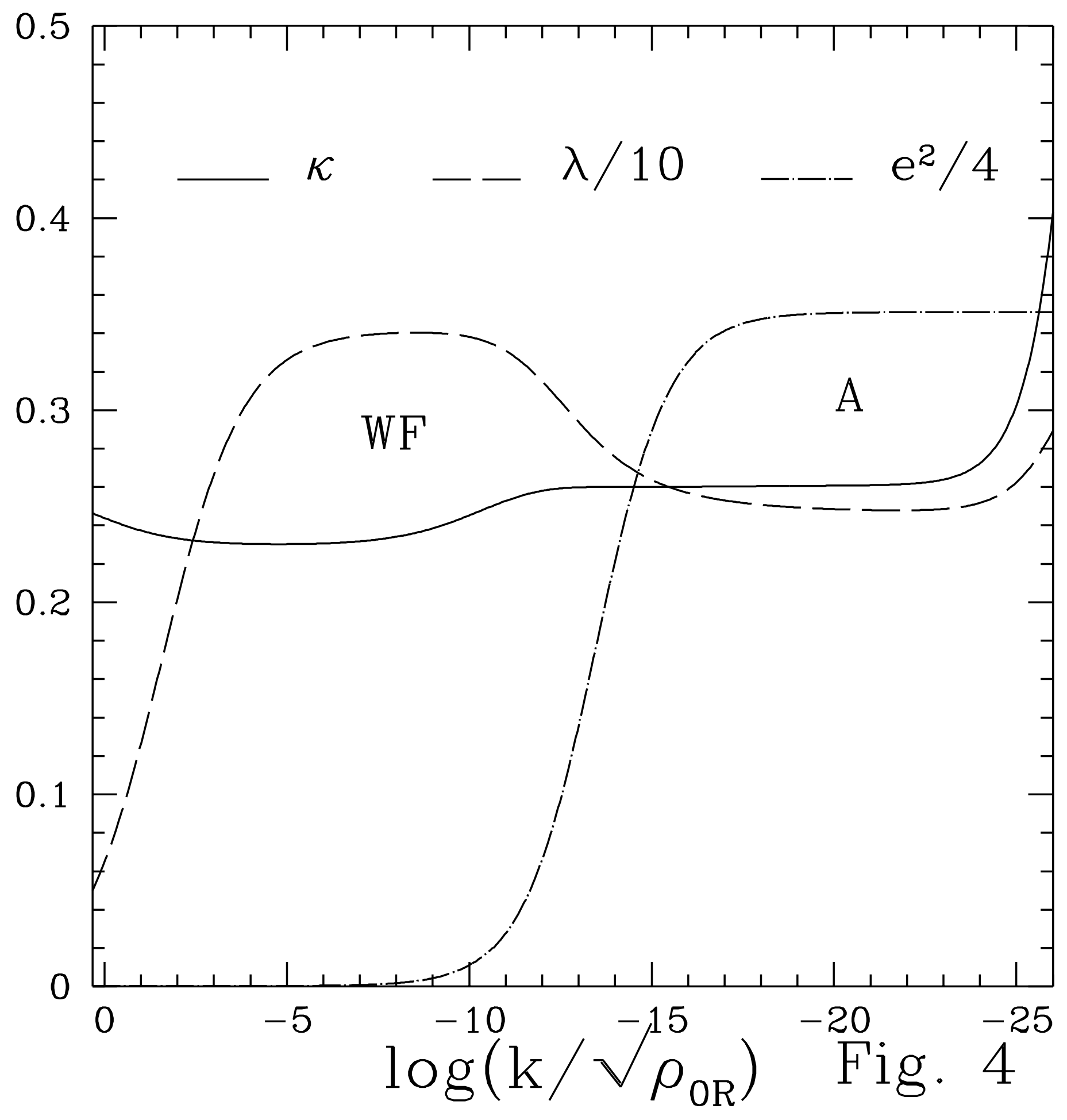




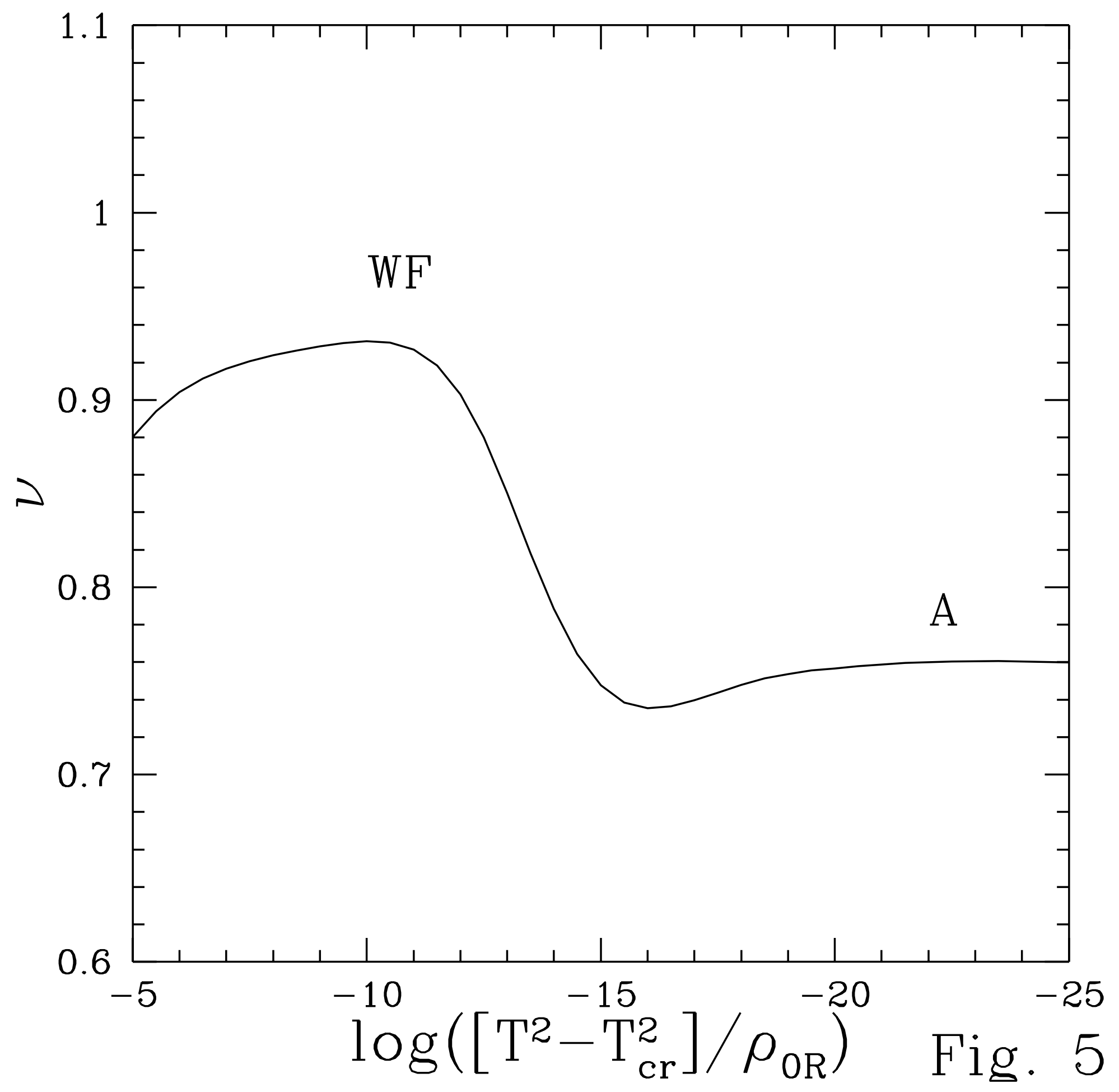




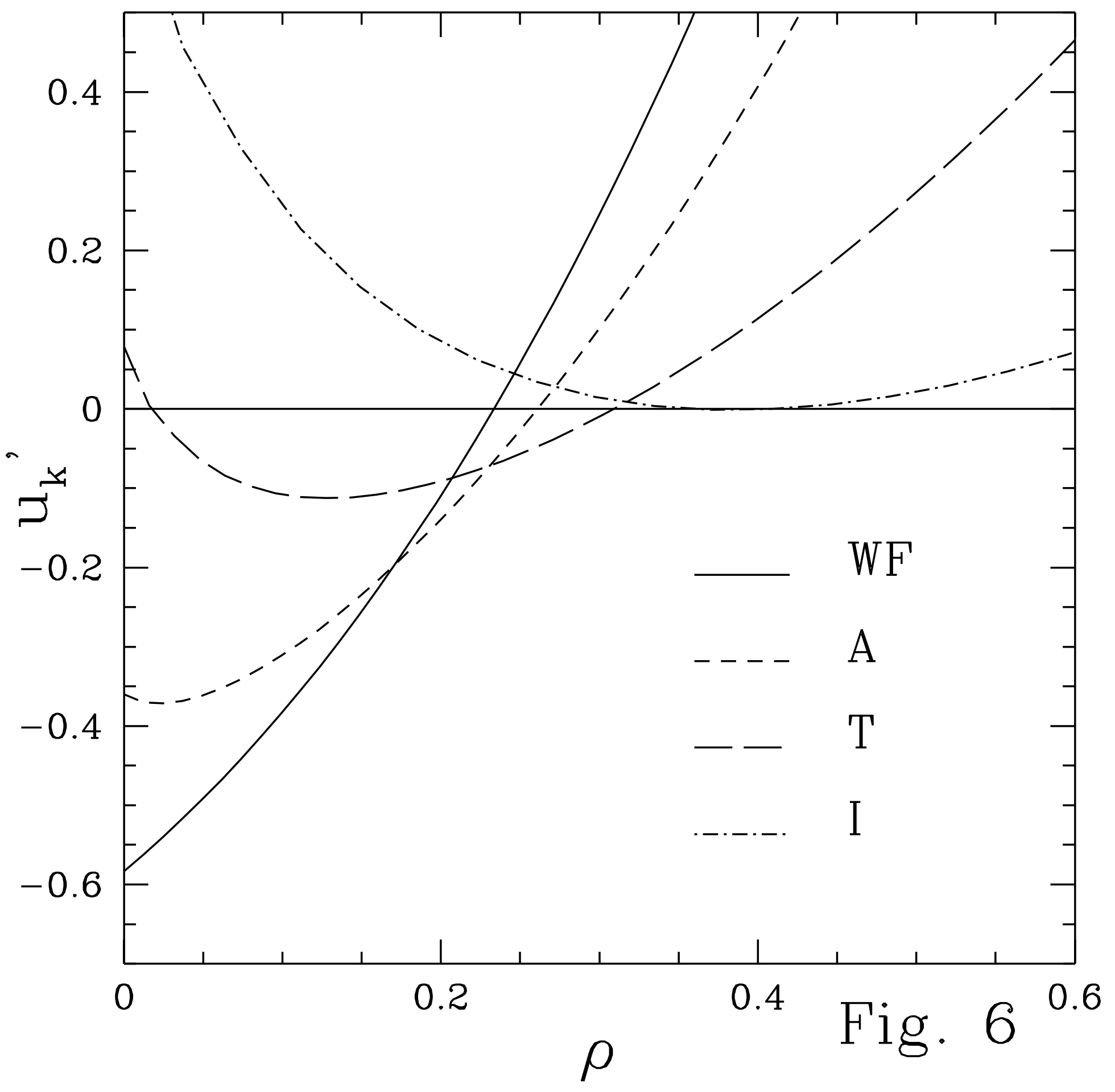




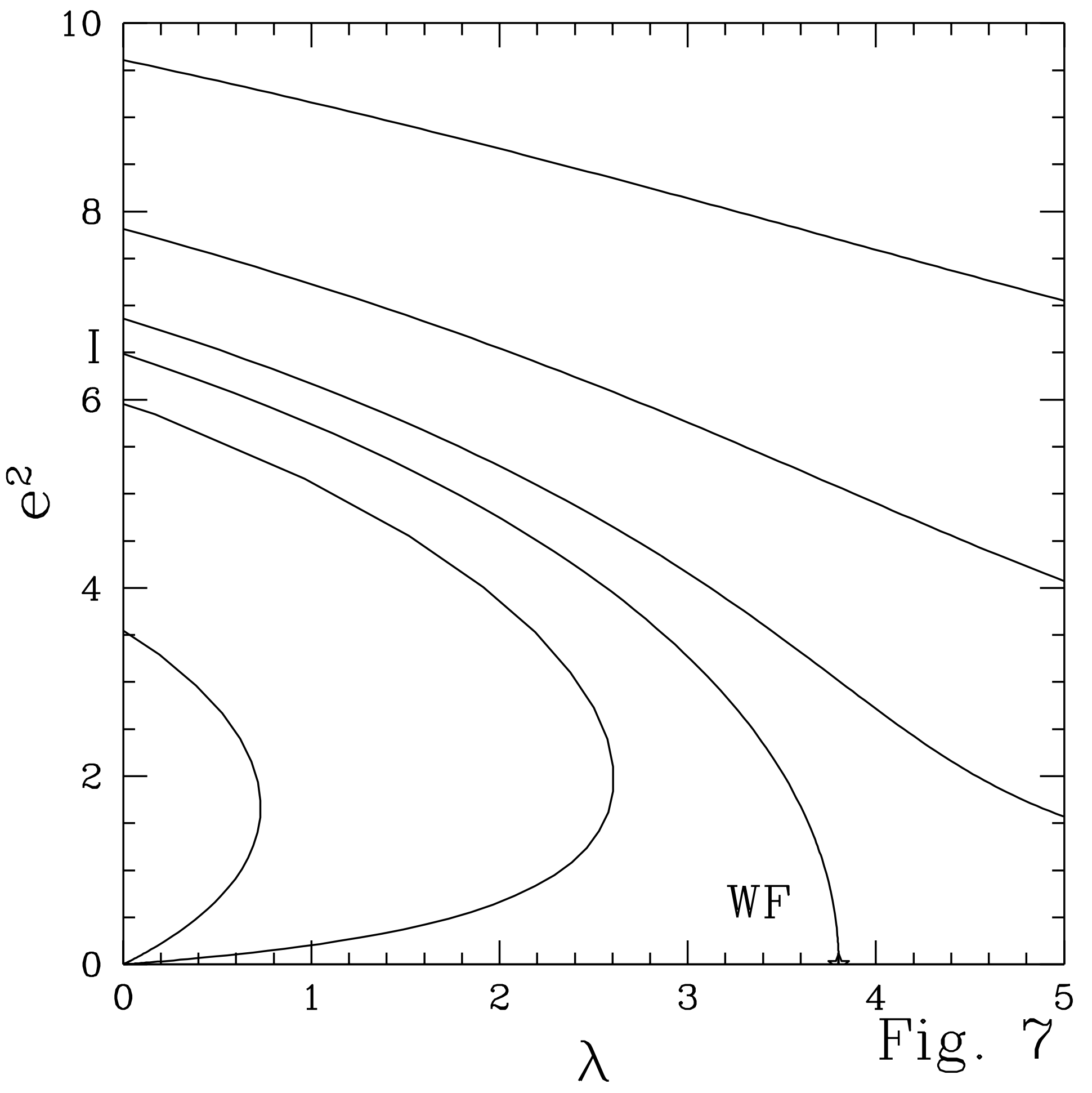




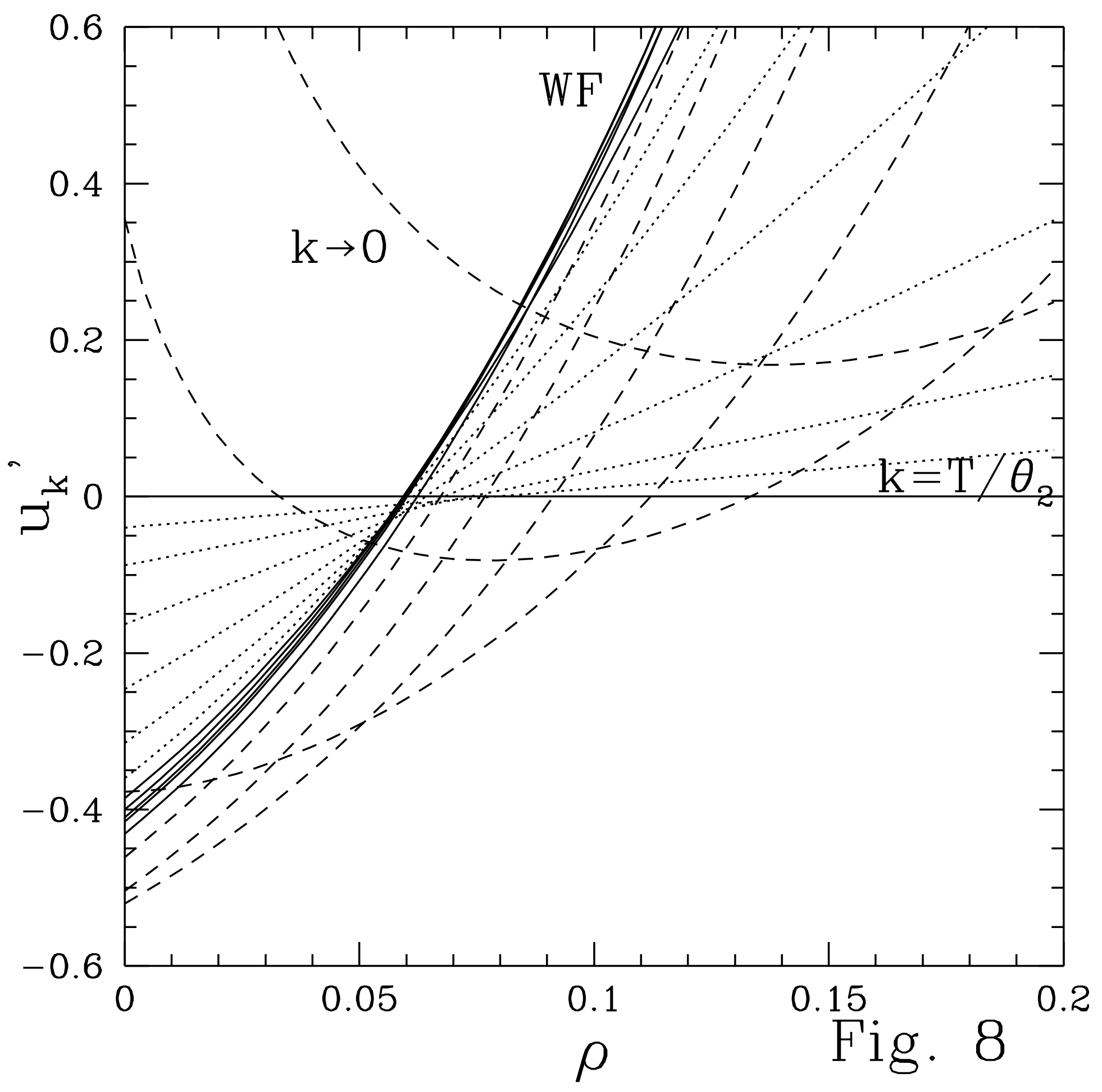




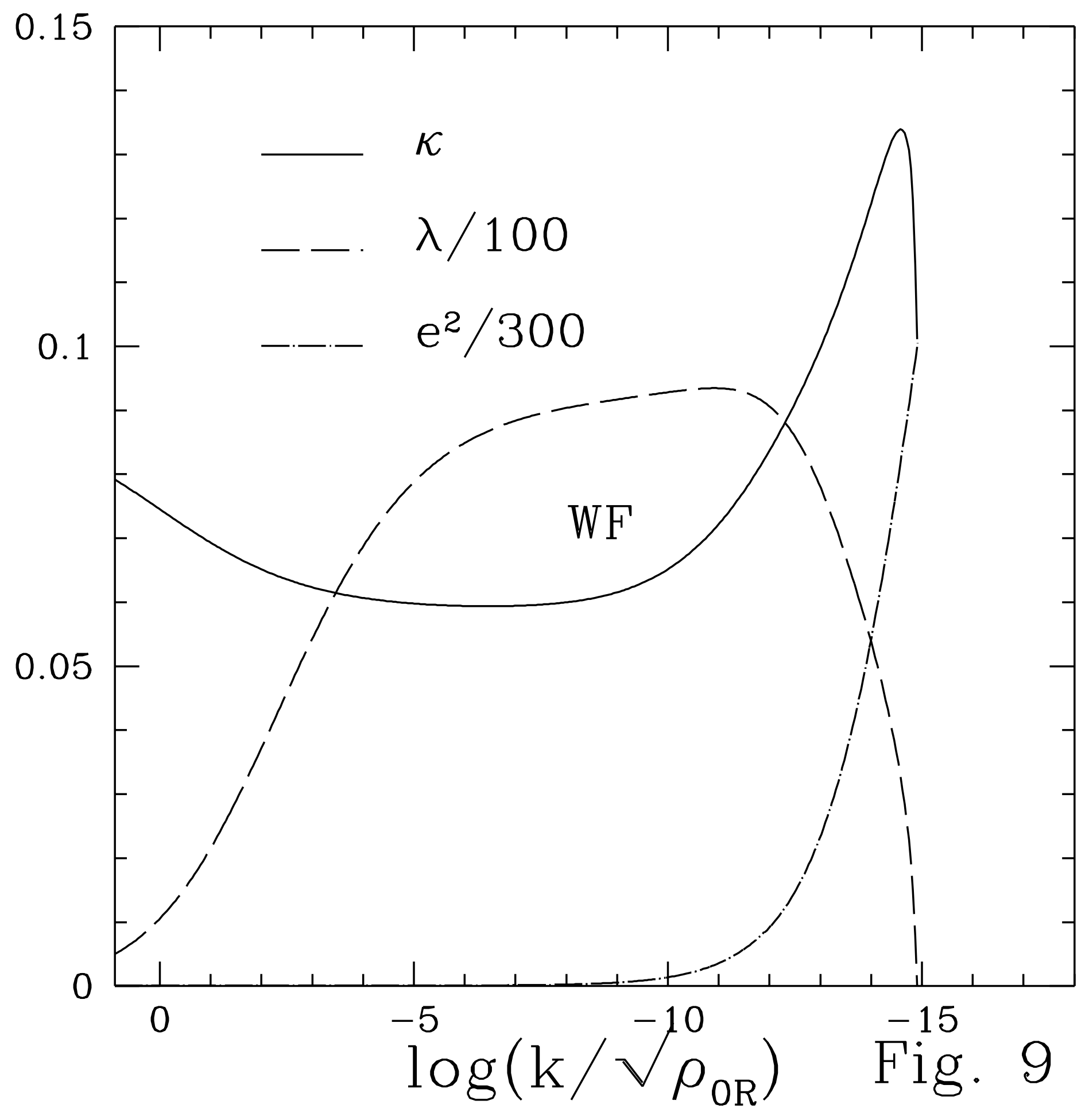




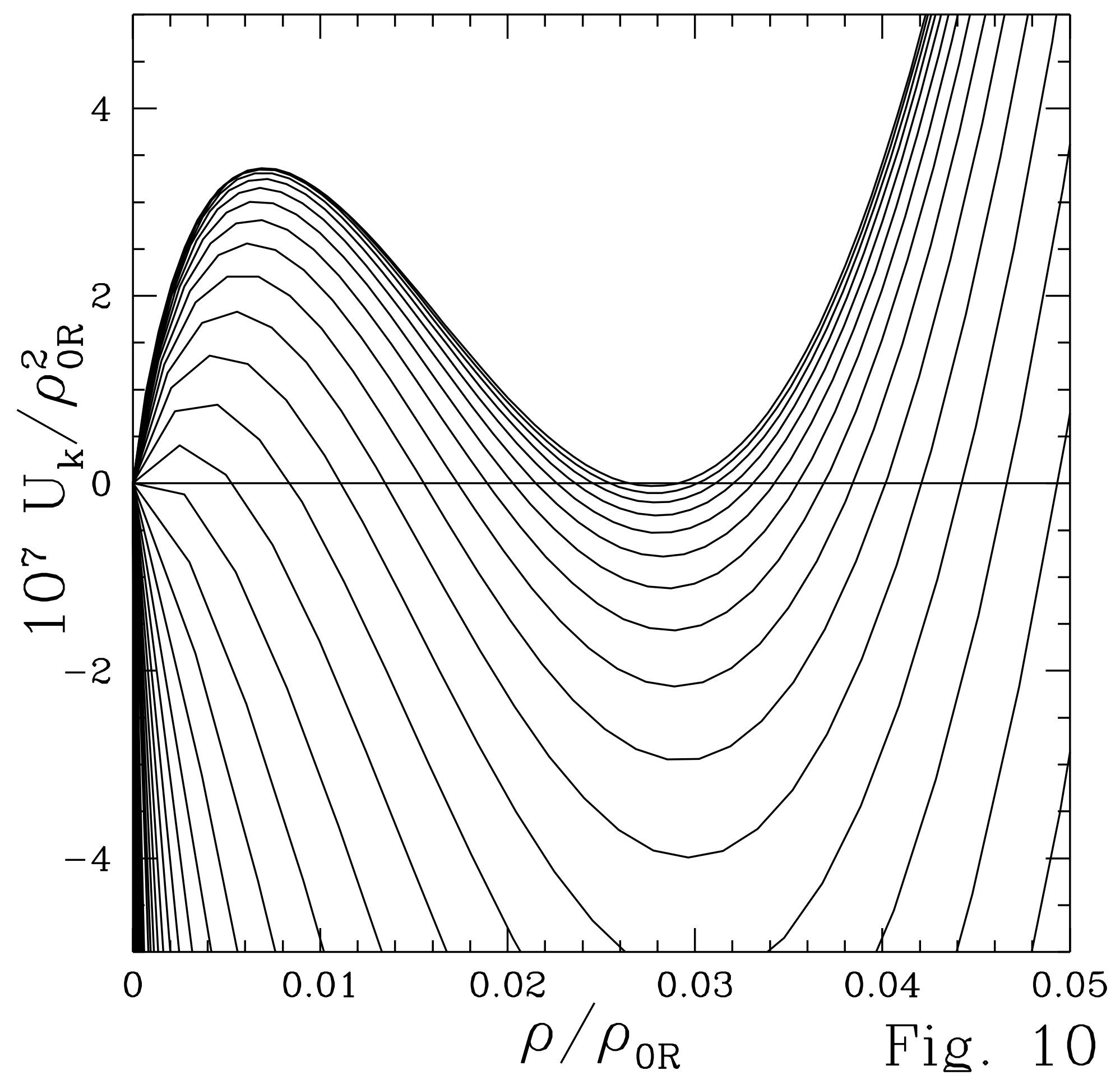




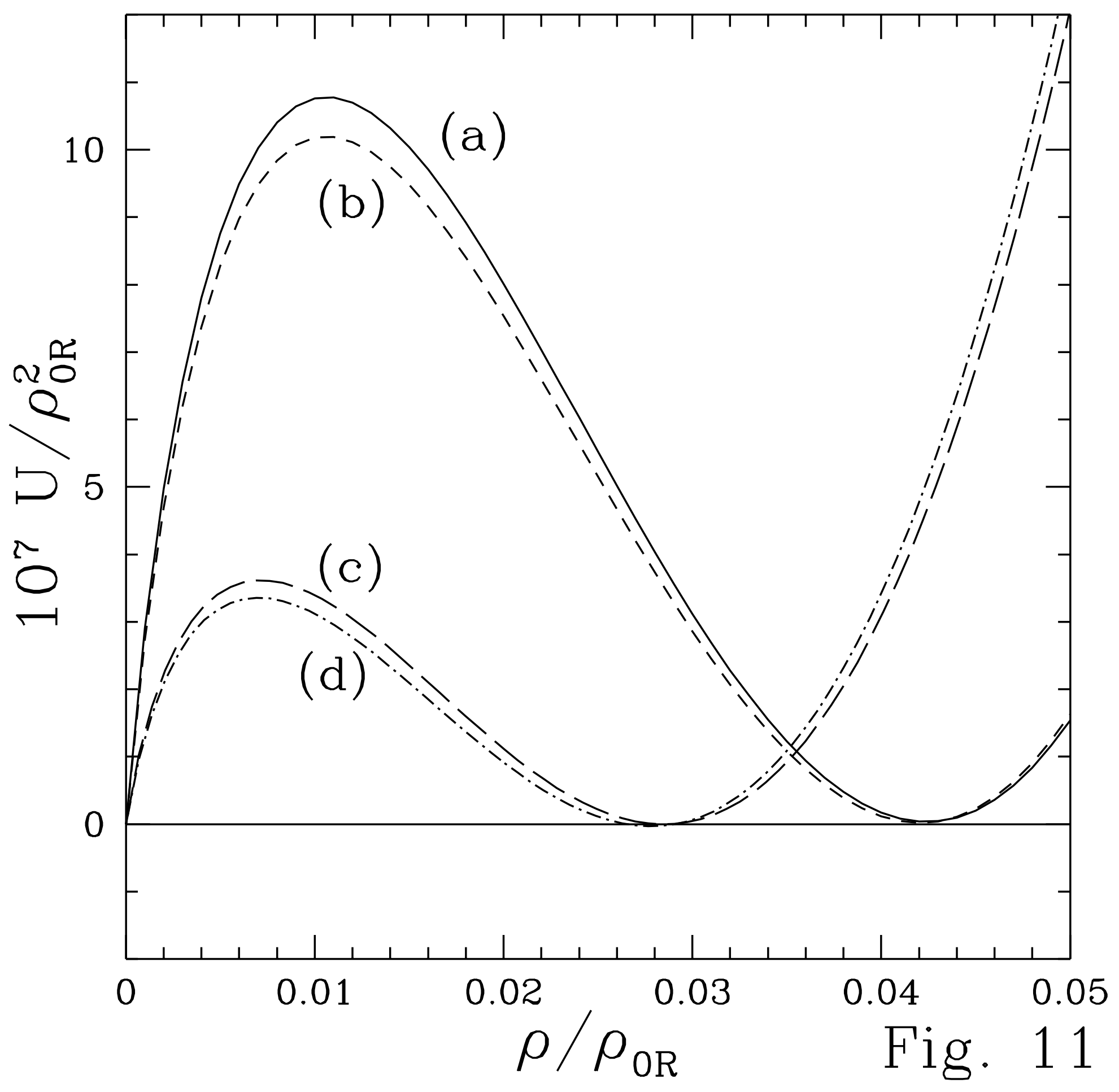




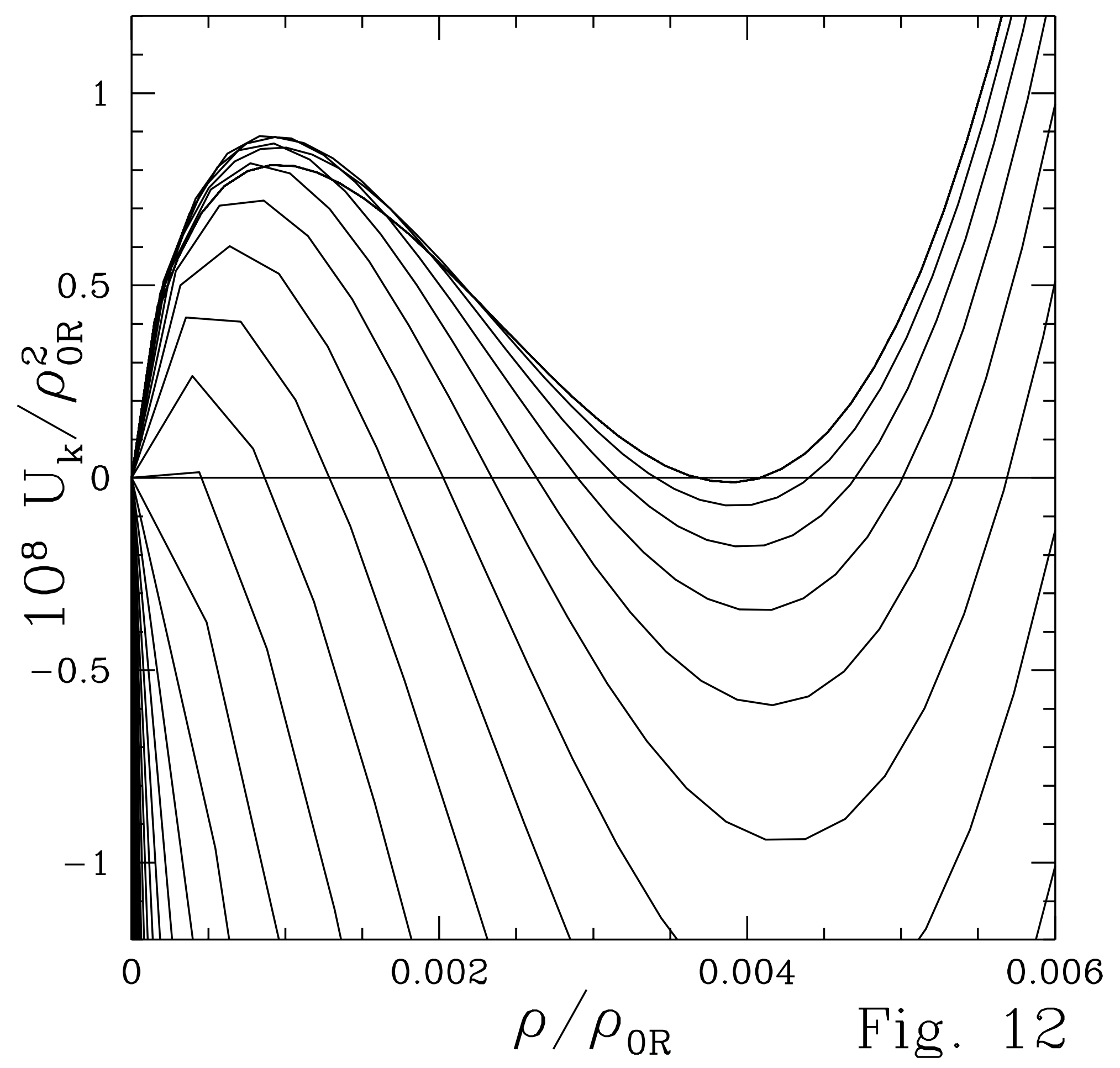




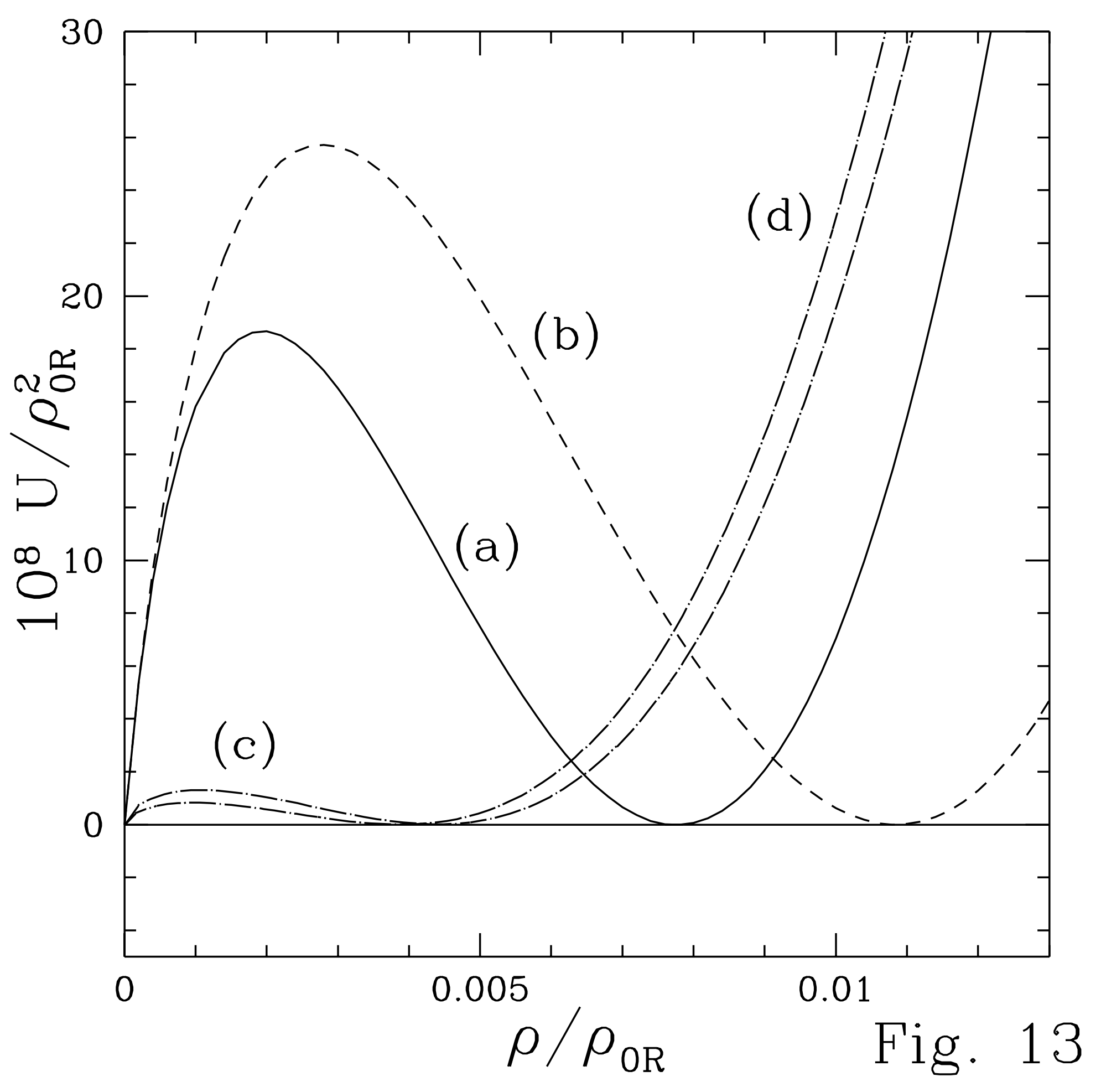




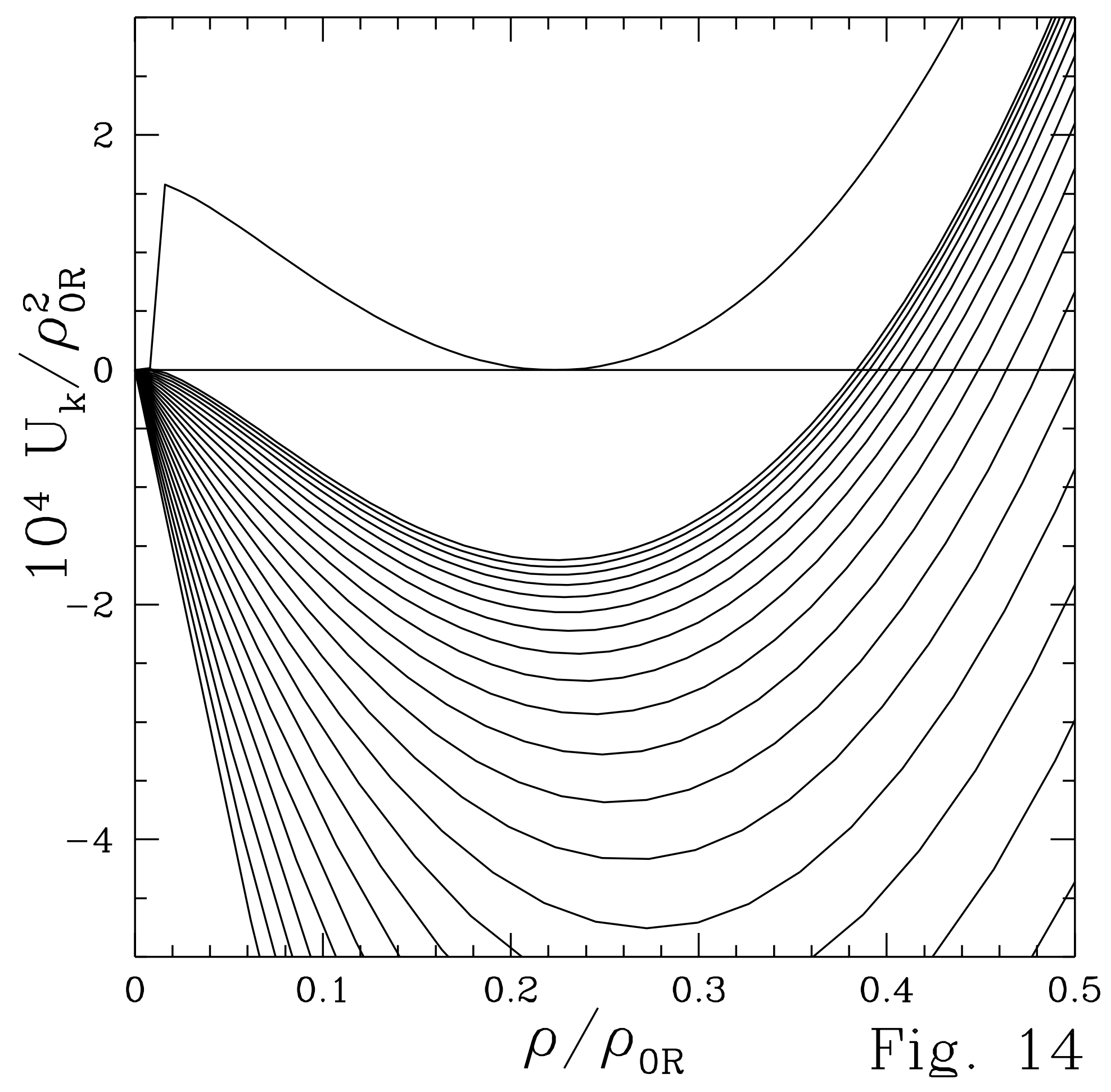




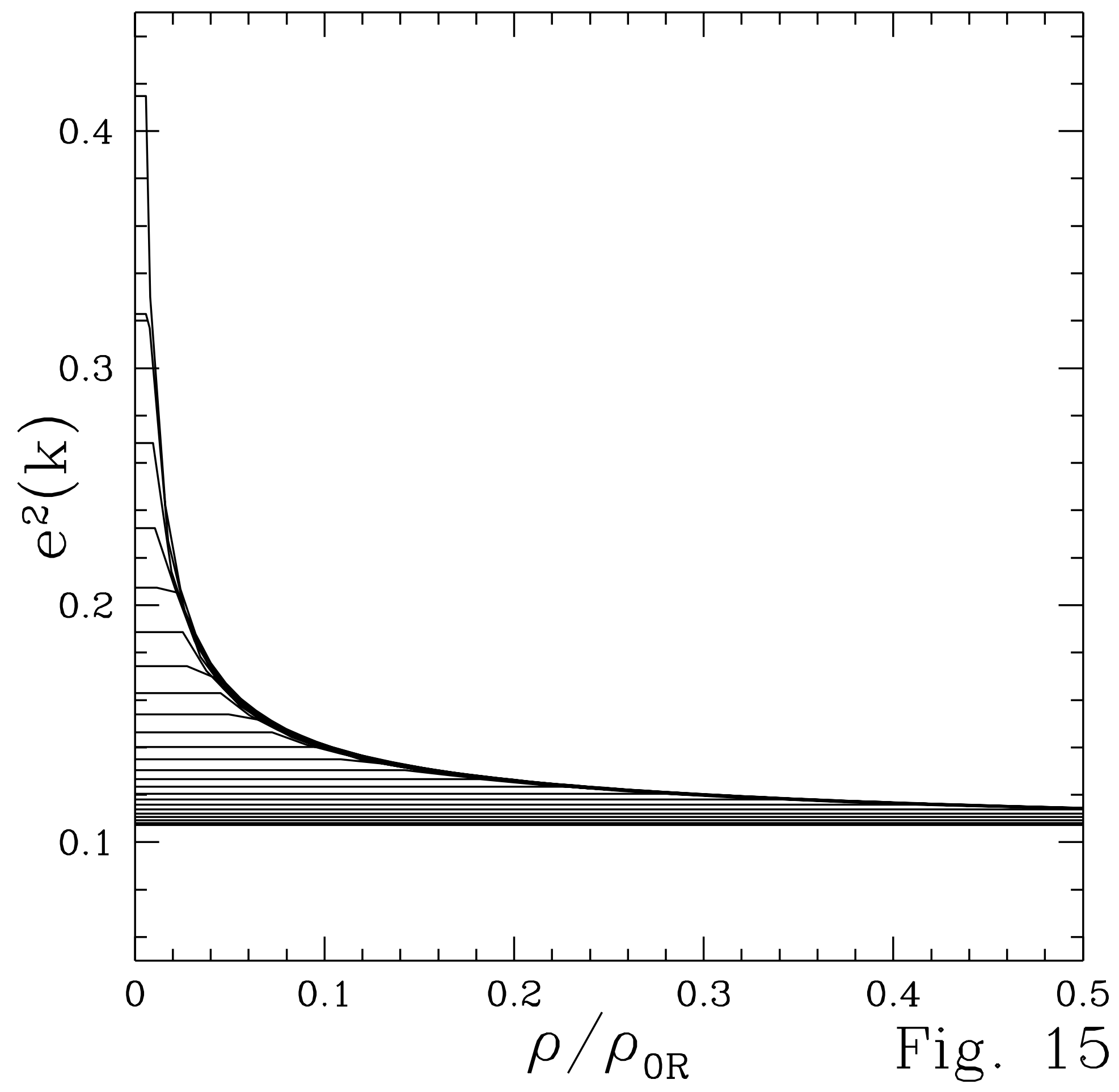




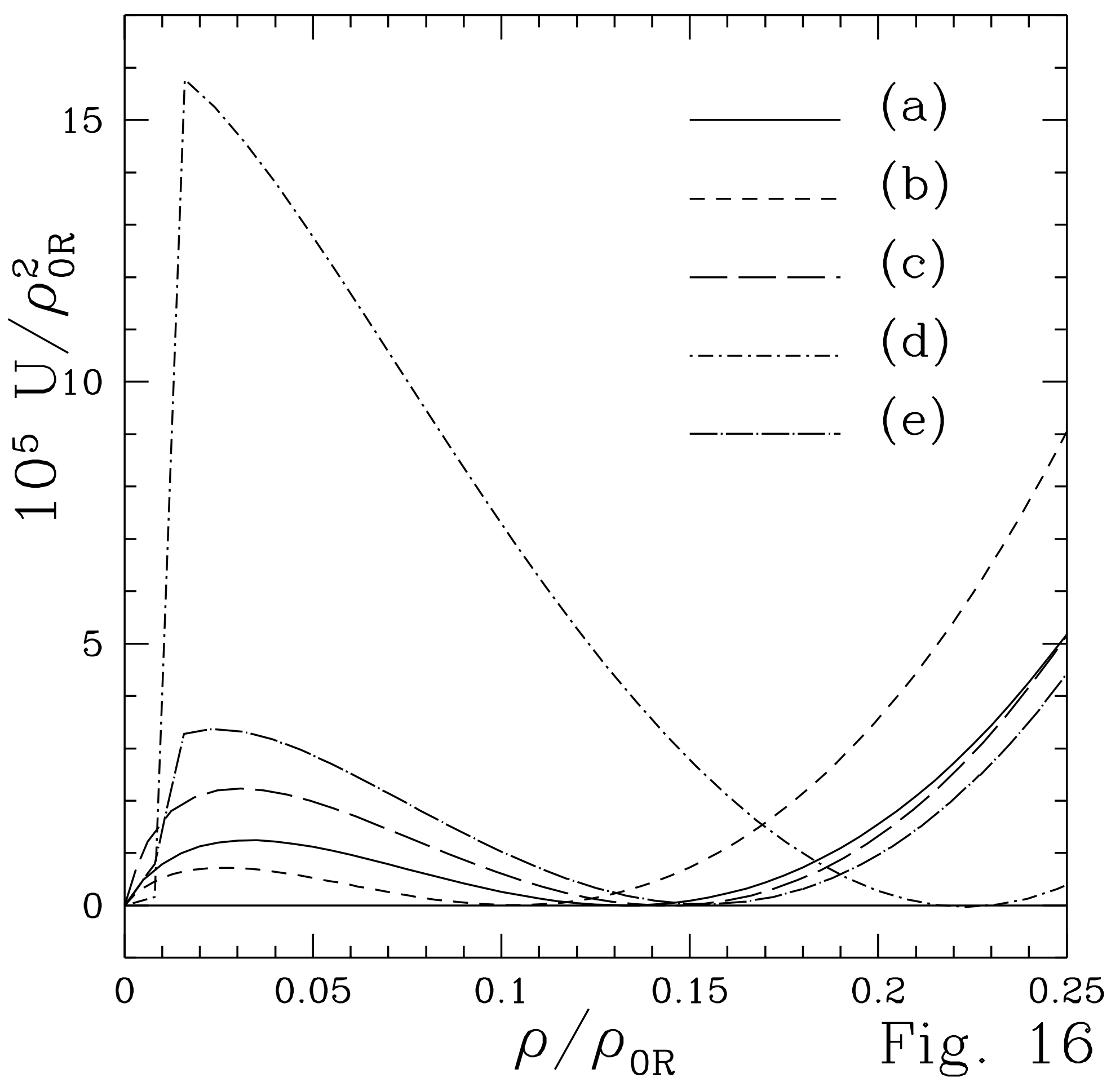




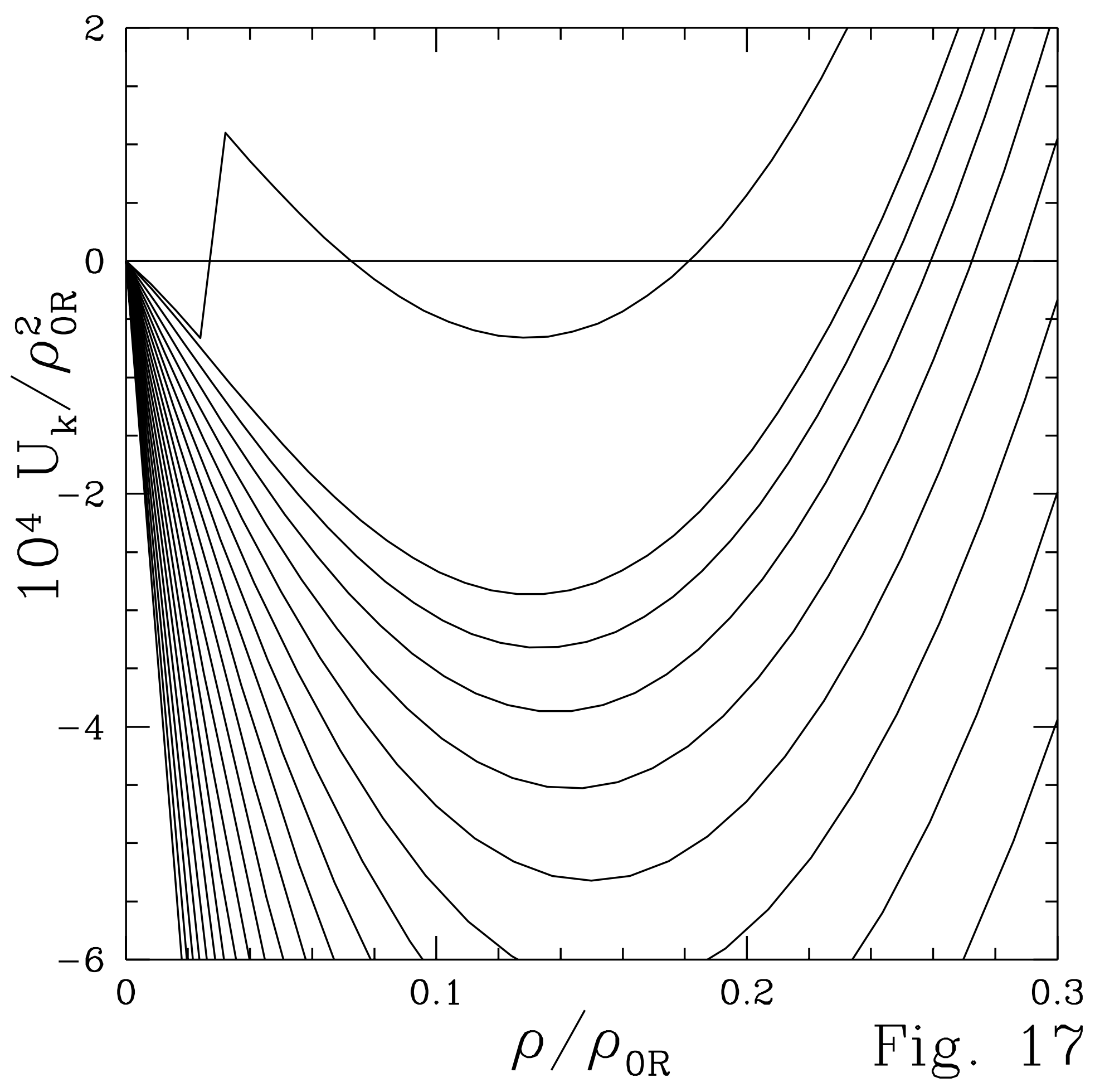

